\title{
Die praktische Anwendung der elektrochemischen Sauerstoffbestimmung im Wasser
}

\section{I: Grundlagen; Langzeitmessung}

\section{Von H. AMBïHL}

(Mitteilung Nr. 89 der Eidgenössischen Anstalt für Wasserversorgung, Abwasserreinigung und Gewässerschutz an der Eidgenössischen Technischen Hochschule in Zürich, Dircktor: Prof. Dr. O. JAAG.)

Manuskript eingegangen am 7. Mai I955

\section{Die methodischen Grundlagen}

Mit der Einführung des sogenannten «Sauerstofflotes» nach TOEDT eröffnete sich der Limnochemie eine schon lïngst erwünschte und gesuchte Möglichkeit, den Sauerstoffgehalt eines Gewässers in jeder beliebigen Tiefe kontinuierlich und ohne besondere Probenahme zu bestimmen. Wohl arbeitet diese Methode in ihrer herkömmlichen Fassung nicht so genau wie die altbekannte Winklersche Bestimmungsweise, doch erlaubt sie durch Verwendung einer sauerstoffempfindlichen Sonde eine sehr rasche Messung, worauf ihre eigentliche Stärke beruht.

Das Prinzip dieser elektrochemischen Methode ist kurz folgendes (ausführliche Angaben dariber siehe ToEDT, I942, I953, 1954, I955; OHLE, I952 und 1953.

Bei Benutzung von Elektrodenpaaren aus Platin-Kadmium, Gold-Kadmium, Gold-Zink usw. macht man die Beobachtung (ToEDT, 1942), dass die Stärke der auftretenden «Korrosionsströme», die zwischen den ausserhalb des Elektrolyten leitend verbundenen Elektroden fliessen, unter anderem von seinem Sauerstoffgehalt abhängen. Das Elektrodenpaar ist ein galvanisches Element, in welchem ein Depolarisationsstrom fliesst, indem die zur Kathode wandernden Wasserstoffionen bzw. der primär entladene Wasserstoff durch den gelösten Sauerstoff oxydiert werden. 
Ohne auf den Reaktionsverlauf näher einzutreten, lässt sich nach TOEDT (I953) die elektrochemische Stromerzeugung prinzipiell durch die Gleichungen darstellen:

$$
\begin{aligned}
\mathrm{O}_{2}+2 \mathrm{H}_{2} \mathrm{O}+2 \mathrm{e}^{-} & =\mathrm{H}_{2} \mathrm{O}_{2}+2 \mathrm{OH}^{-} \\
\mathrm{H}_{2} \mathrm{O}_{2}+2 \mathrm{e}^{-} & =2 \mathrm{OH}^{-} \\
\mathrm{Zn} & =Z \mathrm{nn}^{-}+2 \mathrm{e}^{-}
\end{aligned}
$$

Danach wird einerseits an der Kathode Sauerstoff verbraucht, während gleichzeitig ein alkalischer Hof erzeugt wird, und anderseits geht die $\mathrm{Zn}$ Elektrode allmählich in Iösung; sie wird korrodiert.

( nxere Versuche, die elektrochemische Sauerstoff bestimmung in Respirituminessungen an Wassertieren sowic in physikalischen Experimenten anzuwenden, erzeigten einige Fehlerquellen, die uns veranlassten, diese Methode von einer mehr praktischen Seite her erneut zu prüfen. Wenn TOEDT im Jahre I95 I eine registrierende Anlage zur Bestimmung des $\mathrm{O}_{2}$ Gehaltes im Kesselspeisewasser eines Kraftwerks empfehlen konnte, so darf doch nicht übersehen werden, dass dort für das Funktionicren der Methode in jeder Beziehung die denkbar günstigsten Bedingungen vorherrschen (OHLE, 1953).

Dic Abhängigkeit der Sauerstoff-Diffusionsströme ron verschiedenen, mit dem Sauerstoffgehal t nicht in Zusammenhang stehenden raktoren (Elektrolytgehalt bzw. Leitfähigkeit, Fliessgeschwindigkeit, Bikarbonatgehalt bzw. Karbonathärte) ist es recht eigentlich, was der allgemeinen Anwendbarkeit bis heute hinderlich war. Es zeigte sich indessen (TOEDT, I954), dass durch eine geschickte Auswahl des Kathodenmaterials cincrseits und durch Verkleinerung der Kathode anderseits die störenden Einwirkungen ron Leitfähigkeitsünderungen sowic der Karbonathärte in günstigem Sinne beeinflusst werden kïnnen, so dass zweifellos die Hoffnung bestand, das Gerït, das ja - aus den gïunstigen Lrtcilen ()HLEs zu schliessen - bereits allerbeste Dienste geleistet hat, ciner wirklich allgemeinen Verwendung in Limnologie und Gewässerpraxis zuzuf ühren.

\section{Die Abhängigkeit des Sauerstoffdiff usionsstroms}

von der Strömungsgescbmindigkeit des Elektrolyten; die Form der Katbode

Da um die Kathode herum eine Sauerstoffverarmung eintritt, rermögen Bewegungen des Elektrolyten die Grösse der Sauerstoffdiffusion an diese Elektrode zu verändern. Je mehr man sich der maximalen Diffusion nähert, um so geringer machen sich Strömungsschwankungen geltend. Das bedeutet, dass sich Schwankungen in der Fliessgeschwindigkeit des Wassers, 
sofern dieses eine gleichförmig gerichtete Bewegung ausführt, um so schwächer auswirken, je grösser die mittlere Geschwindigkeit ist (OHLE, 1953; TOEDT, 1953, 1954).

Wir haben mit Hilfe eines Kreislaufsystems (Abb. I; nach JAAG, I955), in dem wir sauerstoffgesättigtes Wasser mit konstanter Geschwindigkeit zirkulieren lassen, die Strömungsabhängigkeit eines Paares von platten-

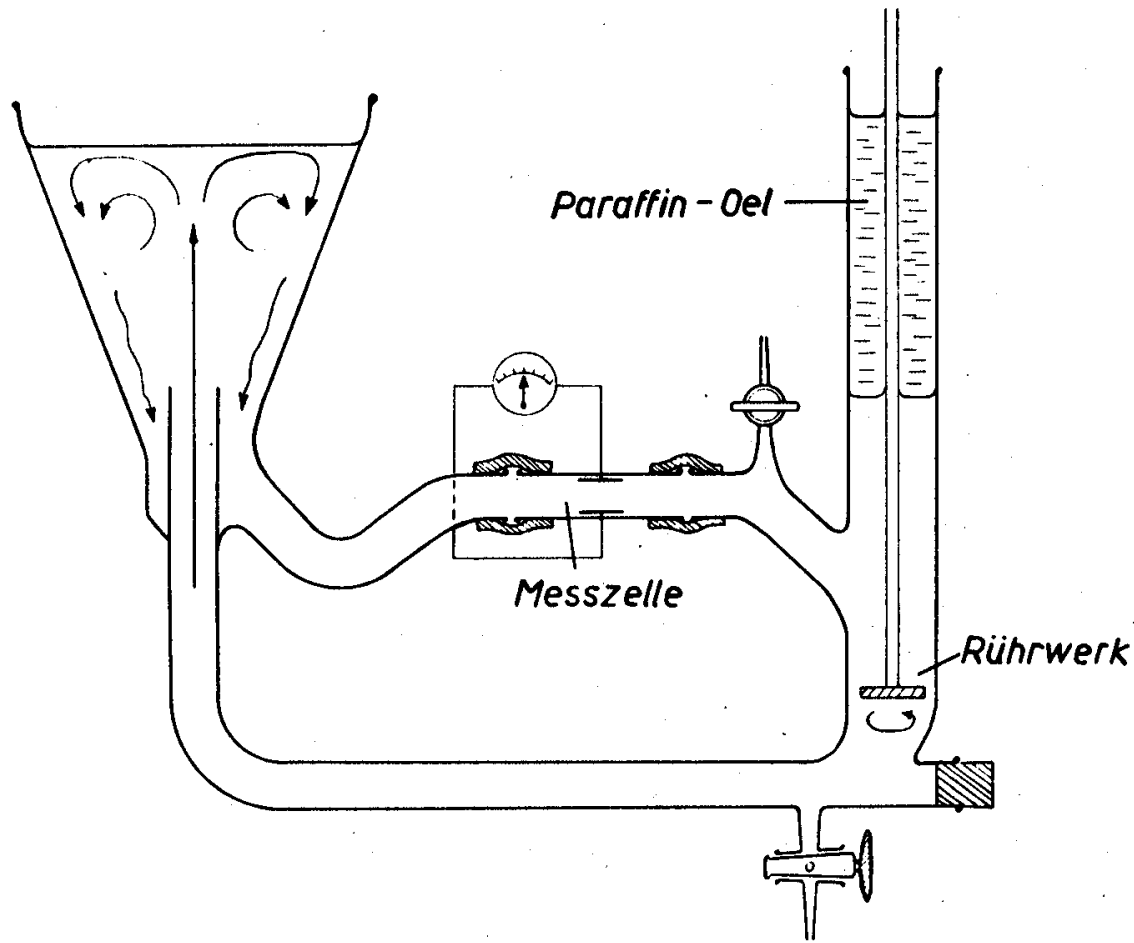

Abb. 1. Umlaufgefäss nach $O$. JAAG (1955), zur Elektrodenprüfung mit karbonatfreiem Wasser verwendet.

förmigen Elektroden geprüft und dabei die Beziehung aus Abbildung 2 erhalten (Elektroden parallel; Kathodenfläche: $3 \times 6 \mathrm{~mm}$, FeingoldBlech; Anode: $5 \times$ Io mm, Zinkblech. Bauweise geht aus der Abbildung hervor).

Diese Kurve bedeutet in praxi, dass wir den Elektrolyten entweder mit beliebiger, aber konstanter Geschwindigkeit an den Elektrodenplatten vorbeifliessen lassen müssen oder versuchen, den «Sättigungswert» bzw. eine möglichst hohe Fliessgeschwindigkeit zu erreichen. OHLE behilft 
sich, indem er sein mit Scheibenelektroden ausgestattetes Sauerstofflot ruckweise schüttelt und so dem Sättigungswert nahekommt.

Strömungstechnisch gesehen gilt es somit, das sauerstoffarme Wasser, das in unmittelbarer Nähe der Kathode entsteht und das sich im Innern der Prandtlschen Grenzschicht dem Zugriff der Strömung weitgehend entziehen kann, fortzuschaffen, damit es sich auf die Sauerstoff-«Nachliefe-

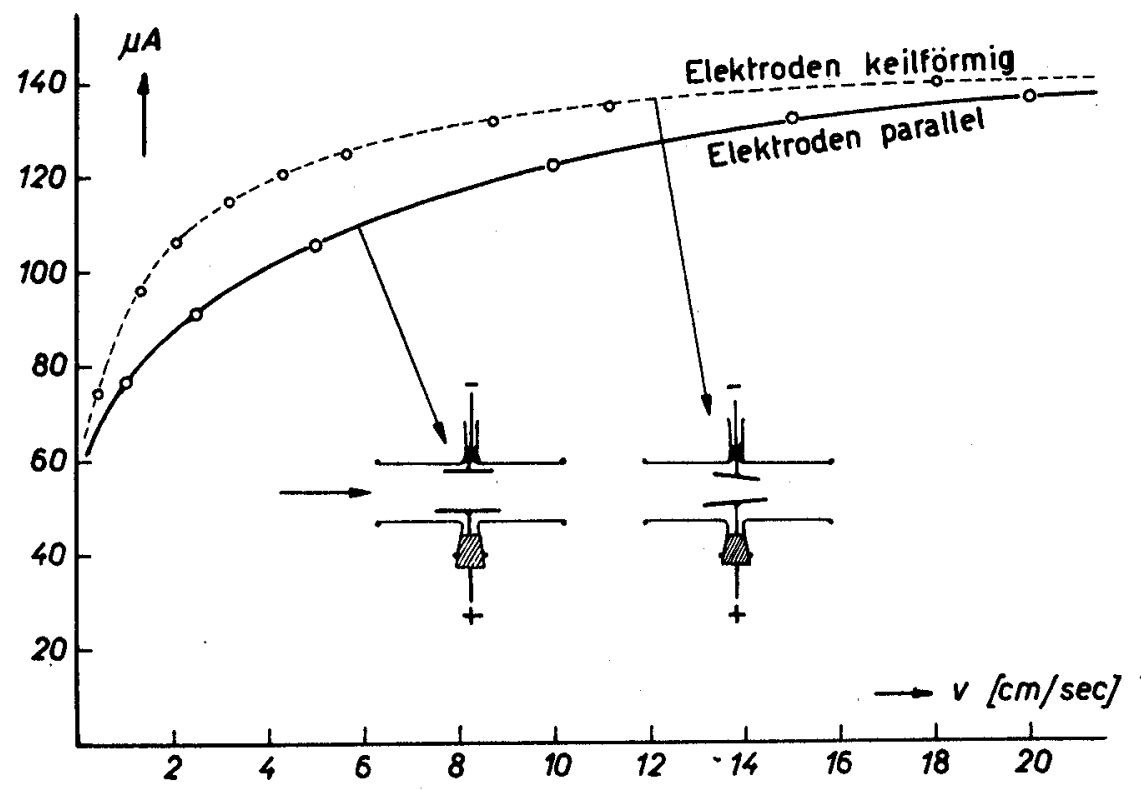

Abb. 2. Abhängigkeit des Sauerstoff-Diffusionsstroms eines Au-Zn-Plattenpaares von der Fliessgeschwindigkeit des Wassers. Die gestrichelte Kurve zeigt die Strömungsabhängigkeit derselben Elektroden, nachdem sie keilförmig gegeneinander verstellt worden waren.

rung" nicht hinderlich auswirkt. Aus der Tatsache, dass sich diese Grenzschicht als rein physikalische Erscheinung überall dort bildet, wo eine Flüssigkeit einen festen Körper umströmt, ergibt sich die Aufgabe, eben diese in unserem Fall hinderliche Schicht möglichst weitgehend zu eliminieren. Gänzlich unterdrücken lässt sie sich jedoch infolge der Kleinheit der Elektroden nicht, aber ihr Volumen kann doch immerhin auf ein Minimum gebracht werden:

Nach PRANDTL (1942) ist die Dicke $\delta$ der Grenzschicht auf einer längs angeströmten Platte $P$ im Abstand $L$ vom Anfangspunkt $A$ an gemessen: 
wobei

$$
\delta=\sqrt{\frac{L}{v} \cdot \frac{\mu}{\varrho}}
$$

$$
\begin{aligned}
& \varrho=\text { spezifisches Gewicht, } \\
& \mu=\text { Viskosität (Zähigkeit) }, \\
& v=\text { Geschwindigkeit. }
\end{aligned}
$$

Je grösser somit $L$ ist, um so dicker wird die Grenzschicht, wobei der Verlauf allerdings nicht linear, sondern mit der Wurzel aus $L$ erfolgt (Abb. 3), und je höher die Geschwindigkeit, um so dünner wird sie. Damit ist bestätigt, dass es sich in unserem Fall wirklich um eine Grenzschichterscheinung handelt, was von TRUEMPLER und ZELLER(I95I) eingehend untersucht worden war.

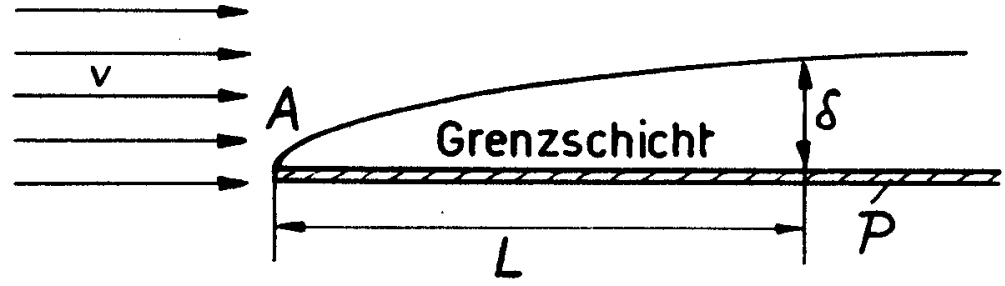

Abb. 3. Schematische Darstellung der Prandtlschen Grenzschicht.

Die gestellte Forderung, das Grenzschichtvolumen möglichst minimal zu halten, kann somit auf zwei Arten angenähert erfüllt werden:

I. Vergrösserung der Gescbwindigkeit. Diese Möglichkeit wird beispielsweise von OHLE ausgenutzt, kann aber im praktischen Gebrauch nicht immer verwirklicht werden, beispielsweise bei Messungen in engen Grundwasserrohren, und stellt zudem an das Material, insbesondere an die Kabel, sehr hohe Anforderungen. Eine zweite Art, die Fliessgeschwindigkeit in Elektrodennähe zu vergrössern, besteht darin, dass man die beiden Elektrodenplatten oder -scheiben keilförmig gegeneinander verstellt. In solchen verengten Fliessquerschnitten wird die Geschwindigkeitsverteilungr spitzer; die Geschwindigkeit in Objektnähe wird erhöht, die Grenzschichtdicke erniedrigt. Der Effekt einer solchen Anordnung unter sonst gleichen Bedingungen geht aus Abbildung 2 hervor.

2. Veränderung der Elektrodenform. Damit die Grenzschichtdicke $\delta$ und damit das Grenzschichtvolumen minimal werden, handelt es sich nach Gileichung (I) darum, die Ausdehnung der Elektrodenfläche in Fliessrichtung möglichst klein zu halten bzw. $L$ zu verkürzen. Man hat demnach beim Bau von Kathoden grosse, zusammenhängende Flächen zu vermeiden, wie sie beispielsweise bei Platten vorhanden sind. 
Demgegenüber war anzunehmen, dass einzelne Drähte oder Drahtgitter den gestellten Bedingungen einigermassen entsprächen. Abbildung 4 gibt die Resultate einiger diesbezüglicher Versuche wieder; in Abbildung 5 sind zudem die dabei geprüften Elektroden dargestellt.

Während Kurve I, gewonnen mit der bereits erwähnten Plattenelektrode, den üblichen Verlauf zeigt, wurde mit einem einzelnen Draht von $0,4 \mathrm{~mm}$ Durchmesser und ungefähr I $3 \mathrm{~mm}$ Länge bereits eine merkliche

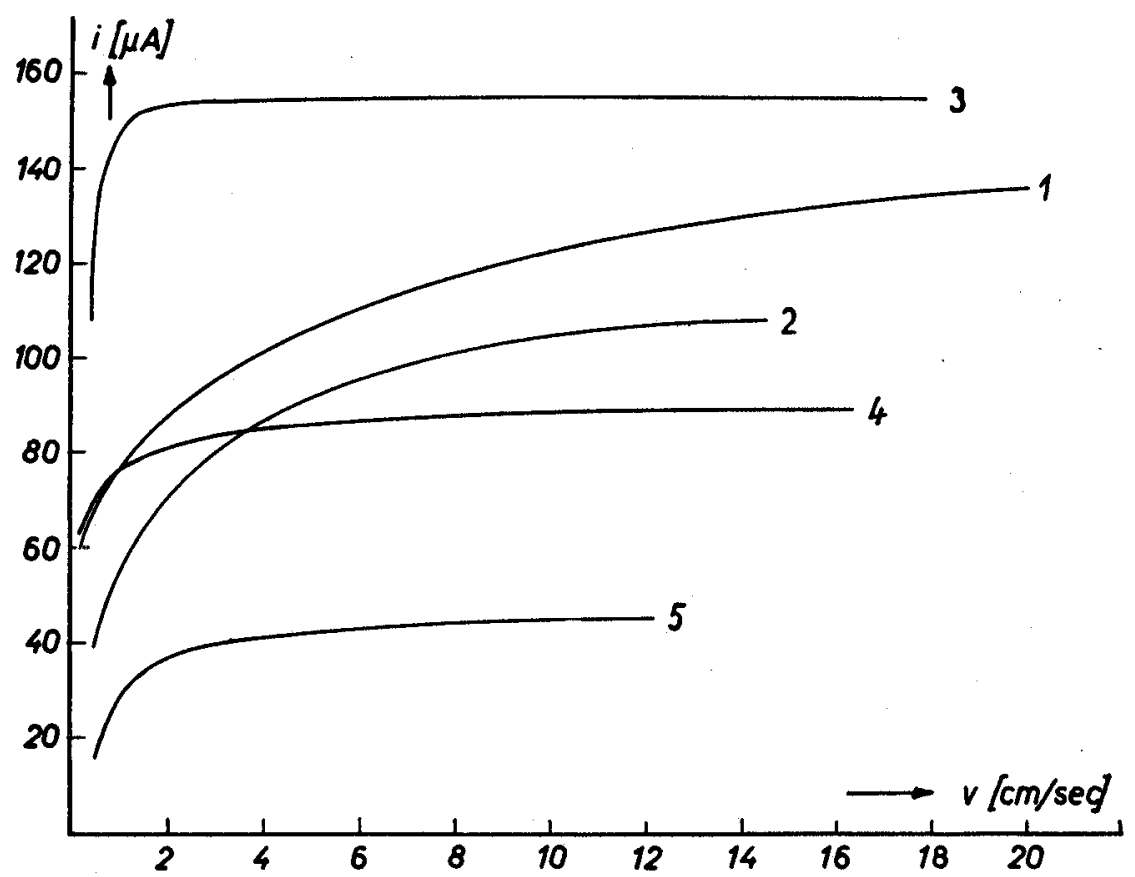

Abb. 4. Strömungsabhängigkeit des Diffusionsstroms einiger Goldkathoden von verschiedener Bauweise. (Die Zahlen beziehen sich auf Abb. 5.)

Verbesserung erzielt, indem sich die $p / i$-Kurve rascher abflacht. Der Anstieg mit steigender Geschwindigkeit erfolgt dagegen noch sehr stetig und erstreckt sich auf ein weites $\boldsymbol{\nu}$-Gebiet. Kurve 3 , erhalten mit einem Gitter von IOO Maschen $/ \mathrm{cm}^{2}$, hergestellt aus $0,25 \mathrm{~mm}$ dickem Draht aus einer Gold-Platin-Legierung im Verhältnis 9:I (HERAEus, Hanau), verläuft hydraulisch ideal, doch ist diese Kathode praktisch nicht verwendbar, da ihre Sauerstoffempfindlichkeit im Diffusionsstromgebiet, das heisst bei den 
normalerweise vorkommenden Sauerstoffgehalten, zu gering ist. Bei sehr geringen Sauerstoffkonzentrationen wäre sie hingegen unter bestimmten Bedingungen brauchbar.

Durch bedeutende Verringerung der Gitteroberfläche und durch eine besondere Verfeinerung in der Formgebung erhielten wir eine Kathode,

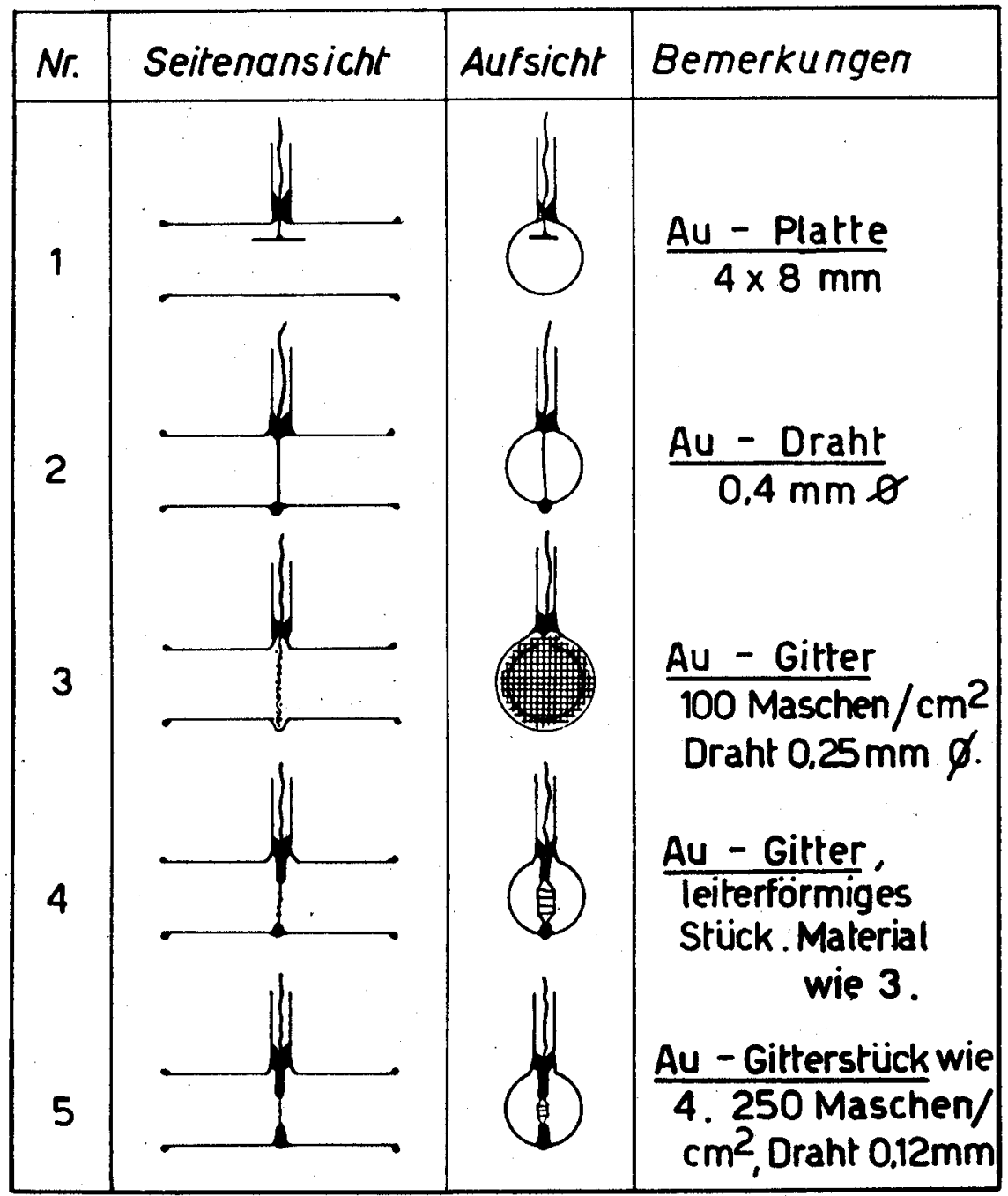

Abb. 5. Darstellung der Kathoden, die auf Strömungsabhängigkeit geprüft wurden (Abb. 4). Die Zinkanoden sind nicht eingezeichnet. 
deren Kurvenverlauf (4) wohl nicht mehr den idealen Verhältnissen von 3 entspricht, aber gegenüber $\mathrm{I}$ und 2 eine deutliche Verbesserung bedeutet. Diese Gitterkathode, das Ergebnis einer lïngeren Entwicklungsreihe, ist auch mit Hinsicht auf ihre Sauerstoffempfindlichkeit als genügend zu betrachten. Wohl ist diese nicht linear ( $v$ gl. dazu OHLE, I953), wenigstens nur in ihrem untersten Bereich, doch stellt sie zwischen allen in Frage stehenden Komponenten eine Kompromisslösung dar, die einigermassen befriedigen dürfte, wären nicht noch weitere, später zu erörternde Punkte zu berücksichtigen. Dic letzte Kathode schliesslich, eine Gold-Platin-Kleinstelektrode ( $(\xi)$, wurde mit Hinsicht auf Feldmessungen entwickelt. Da hier

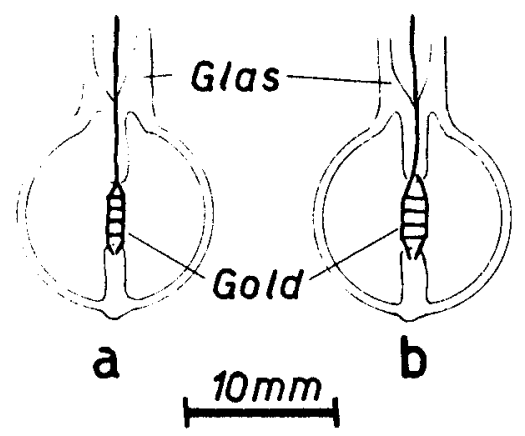

Abb. 6. Querschnitt durch zwei Gitterkleinstkathoden (nähere Erläuterung im Text). Drahtdicke 0,12 mm; Maschenweite des Goldgewebes $0,63 \mathrm{~mm}=250$ Maschen $/ \mathrm{cm}^{2}$.

ganz besonders auf Linearität der Sauerstoffempfindlichkeit innerhalb des normalen Bereichs, möglichste Unabhängigkeit von Leitfähigkeitsschwankungen und möglichste Strömungsunabhängigkeit geachtet werden muss, wurde die Gitterkathode 4 mehr oder weniger unverändert übernommen und lediglich stark verkleinert. Während völlige Sauerstofflinearität zwischen 0 und $8,5 \mathrm{mg} / \mathrm{l}$ erreicht und auch die Strömungsabhängigkeit auf ein erträgliches Mass herabgemindert werden konnte (Abb. 4, Kurve 5), scheint es indessen kaum möglich, die gewünschte Leitfähigkeitsindifferenz zu erreichen (siehe dazu Abb. 8). Man hat sich somit auch hier mit einem Kompromiss zu begnügen, daneben aber, oder gerade deshalb, mit allen Mitteln zu versuchen, aus den gegebenen Materialkomponenten, wie Maschenweite des Gewebes, Drahtdurchmesser usw., jene Kombination zu finden, die die besten hydraulischen Eigenschaften besitzt. 
Dass man hier auch sehr geringfügige Änderungen in der Kathodenform zu berücksichtigen hat, zeigt der folgende Vergleich zwischen zwei ähnlichen Gitter-Kleinstkathoden. Während die beiden Längsstreben der Kathode $a$ in Abbildung 6 ursprünglich zwei nebeneinanderliegende Gitterdrähte waren, die durch Verschmelzen der Querverbindungen miteinander verbunden wurden, entstand Kathode $b$ wohl auf ähnliche Weise, doch wurden hier als Längsstreben der erste und dritte Paralleldraht benützt,

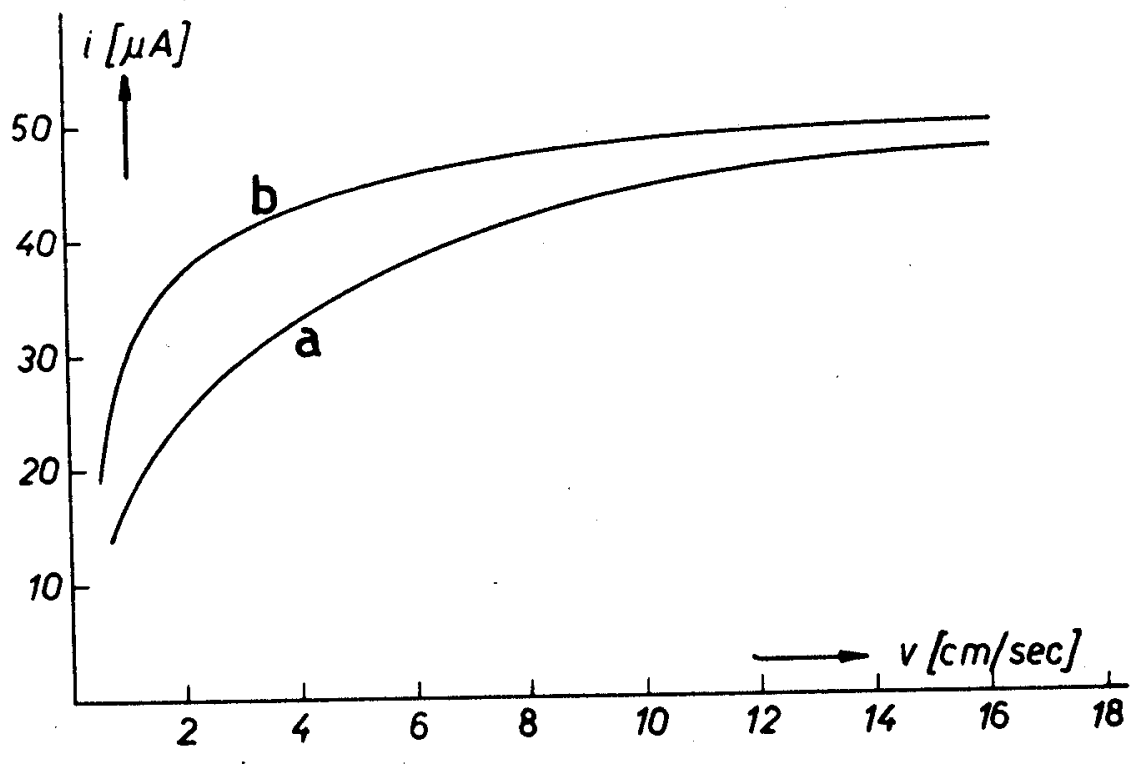

Abb. 7. Dic Strömungsabhängigkeit der beiden Kleinstkathoden in Abbildung 6 .

während der zweite ausgelassen wurde. Dadurch entstand ein leiterförmiges Gitterstück mit doppelt so grossen Maschen wie bei $a$, was sich strömungstechnisch recht bedeutsam auswirkt. Zeigt Kathode $a$ mit steigender Strömungsgeschwindigkeit einen relativ langsamen Anstieg des Sauerstoff-Diffusionsstroms (Abb. 7), so ist er bei $b$ vorerst - bis auf etwa $3 \mathrm{~cm} / \mathrm{s}$ - sehr steil, während der nachfolgende Bereich eine wesentlich geringere Änderung erfährt. Dass unter Umständen sogar die Form der beiden gläsernen Stützen einen Einfluss auf die Strömungsabhängigkeit einer Kleinstkathode ausüben kann, sei hier nur beiläufig erwähnt.

Jede Verkleinerung der Kathode hat zwangsläufig eine vermehrte Strömungsabhängigkeit zur Folge.' Da es jedoch - wenigstens bei der feld- 
mässigen Sauerstoffmessung - trotzdem wünschenswert ist, sie möglichst klein zu gestalten, stellt sich die Forderung, jede gebotene Möglicbkeit auszuscböpfen, um die in Kauf genommene Verschlechterung wieder einigermassen zu kompensieren. Die kürzlich von TOEDT und PETSCH (1955) beschriebene neueste Ausf ührung des Sauerstofflotes ist ein Versuch in dieser Richtung, indem nach dem Prinzip des verengten Kanals (Beispiel siehe Abb. 2!) die Fliess-

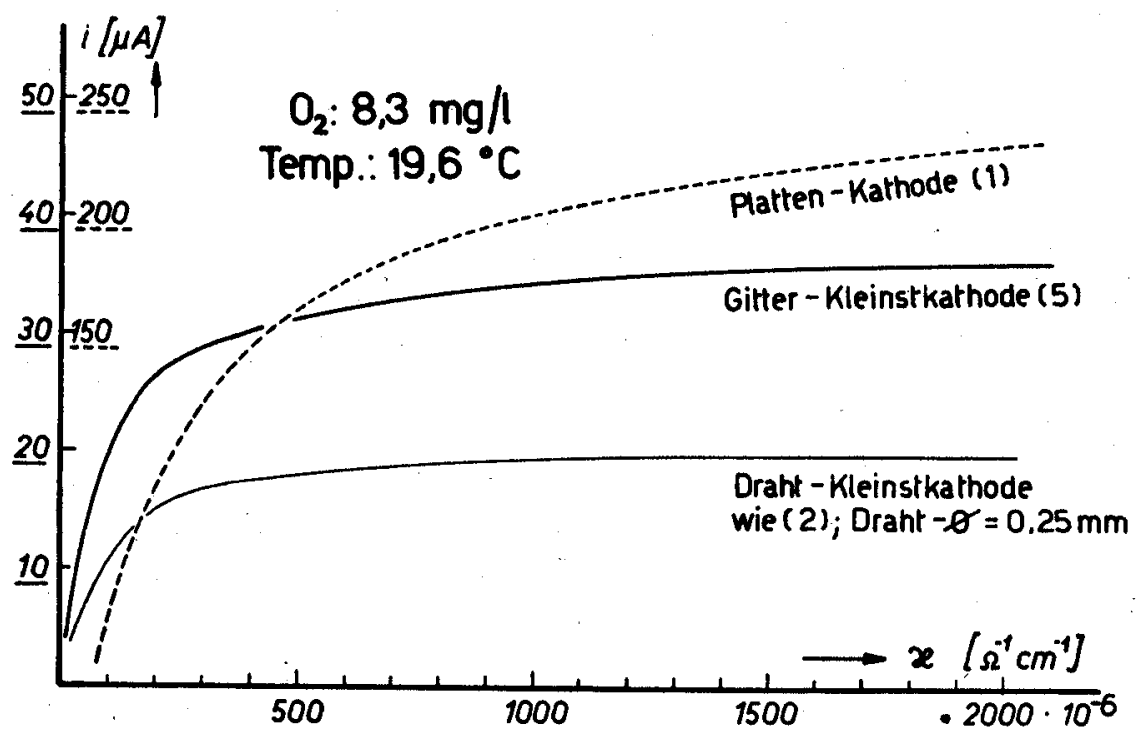

Abb. 8. Bexinflussung des Diffusionsstroms durch die Leitfähigkeit des Wassers. Der Strom der Plațtenkathode (Abb. 5, 1) ist in einem fünffach verkleinerten Maßstab aufgetragen.

geschwindigkeit des Elektrolyten erhöht wird. Obwohl nach dem Urteil der Autoren eine bemerkenswerte Verbesserung gegenüber dem Ohleschen Sauerstofflot erzielt wurde, scheint damit unseres Erachtens das Optimum noch nicht erreicht.

2. Die Abbängigkeit des Sauerstoffdiffusionsstroms von der Leitfäbigkeit des Elektrolyten

Wie TOEDT zeigte, ist die Abhängigkeit des Sauerstoffdiffusionsstroms von der Leitfähigkeit des Elektrolyten bei grossen Elektroden erheblich und kann dort unter Umständen eine brauchbare Sauerstoffmessung verhindern. Mit steigendem Salzgehalt vermindert sich diese Abhängigkeit, 
das heisst, der Diffusionsstrom nähert sich einem konstanten Endwert, der, wie Versuche von TOEDT und Mitarbeiter ergaben, durch die Oberfläche der Elektroden gegeben ist. Verkleinert man nun die Elektrodenfläche, so verringert sich wohl der Strom proportional dazu, doch der Widerstand zwischen den Elektroden verändert sich nach der folgenden Funktion:

wobei

$$
R_{E l}=\frac{1}{x} \cdot \frac{h}{\sqrt{Q_{1} Q_{2}}},
$$

$R_{E l}=$ Elektrolytwiderstand,

$Q_{1,2}=$ Fläche der Elektroden 1 und 2,

$h$. = Abstand der Elektroden,

* = Spezifische Leitfähigkeit (nach VetTer aus ToEDT, 1954)

Somit kommt eine Verkleinerung der Elektrodenfläche im Effekt einer Erhöhung des Elektrolytwiderstandes gleich. Dadurch bietet sich die Möglichkeit, durch genügende Verringerung der Elektrodenfläche in einen Empfindlichkeitsbereich zu gelangen, in welchem sich Leitfähigkeitsänderungen nicht mehr auswirken, was bedeutet, dass man die Kathodenfläche so klein zu wählen hat, dass der «kritische Leitfähigkeitswert», das heisst jener Wert von $\varkappa$, bei dem die $\varkappa / i$-Kurve horizontal wird, unter der in natürlichen Wässern vorhandenen Leitfähigkeit liegt.

Diesen Idealfall zu erreichen, wäre auch aus einem andern, bereits kurz erwähnten Grunde ausserordentlich erwünscht:

Wie OHLE (1953) und TOEDT (1954) darlegten, ist bei grösseren Kathoden, beispielsweise mit einer geometrischen Oberfläche von $0,5 \mathrm{~cm}^{2}$, nur in den untersten Sauerstoff konzentrationen ein lineares Sauerstoff-StromVerhältnis festzustellen. Je mehr man die Kathodenfläche verringert, um so grösser wird dieser lineare Bereich. Wäre es demnach möglich, eine in strömungstechnischer Hinsicht befriedigende Kathode von sehr kleiner Oberfläche zu schaffen, so wären damit zwei grundlegende Forderungen erfüllt:

\section{Unabbängigkeit von Leitfäbigkeitsscbwankungen,}

2. Lineares Sauerstoff-Strom-Verbältnis.

Wie die Erfahrung lehrt, ist indessen die erwünschte Unabhängigkeit vom Elektrolytgehalt des Wassers nicht gänzlich erreichbar. Unsere Elektrodenprüfungen haben jedenfalls in keinem Fall eine pöllig borizontal perlaufende $x / i$-Kurpe ergeben, sondern bestenfalls eine Kurve, die sich asymptotisch einem Endwert nähert, wobei immerhin die Beobachtung bestätigt wurde, dass sich der steil ansteigende Schenkel der Kurve mit kleiner werdender Kathodenfläche gegen die geringere Leitfähigkeit zu verschiebt. 
Es dürfte somit unter praktischen Umständen kaum möglich sein, eine pon der Leitfäbigkeit des W assers völlig unabbängige Elektrode zu scbaffen, sondern wir können sie bloss derart gestalten, dass ihr Arbeitsbereich im flachen Kurventeil liegt, wodurch sie auf Leitfähigkeitsschwankungen wenigstens nurmehr schwach empfindlich wird. Wie Abbildung 8 zeigt, kann unter entsprechenden Umständen diese Empfindlichkeit genügend gering werden, so dass die Leitfähigkeitswerte innerhalb ein und derselben Untersuchungsserie, beispielsweise innerhalb eines einzelnen Seeprofils, vernachlässigt werden könnten. Nicht vernachlässigen dürfte man sie dagegen bei Untersuchungen innerhalb verschiedener Gewässer. Das hätte zur Folge, dass parallel zu jeder elektrochemischen Sauerstoffmessung mindestens eine Leitfähigkeitsbestimmung oder eine Sauerstoffbestimmung nach WINKLER (Kombinationsverfahren nach OHLE) ausgeführt werden müsste.

\section{Der Einfluss des Bikarbonatgebaltes $b z w$. der Karbonatbärte auf die elektrochemische Sauerstoffmessung}

Das grösste Hindernis für eine allgemeine Anwendbarkeit der elektrochemischen Sauerstoffmessung bildete bis vor kurzem der in natürlichen Gewässern stets vorhandene wechselnde Gehalt an Kalzium- und Magnesium-Bikarbonat. Wie aus dem Reaktionsverlauf an der Kathode ersichtlich ist, bildet sich hier im Verlaufe der Messung ein Úberschuss an Hydroxylionen. Dadurch entsteht um die Kathode herum ein sauerstoffarmer, alkalischer Hof, in welchem infolge der Anwesenheit von Kalziumionen unlösliches, kristallines Ca-Karbonat auf die Kathodenoberfläche niedergeschlagen wird:

$$
\begin{aligned}
& \mathrm{HCO}^{\prime}{ }^{\prime}+\mathrm{OH}^{\prime}=\mathrm{H}_{2} \mathrm{O}+\mathrm{CO}_{3}{ }^{\prime \prime}, \\
& \mathrm{CO}_{3}{ }^{\prime \prime}+\mathrm{Ca}=
\end{aligned}
$$

Durch diese Kalkinkrustation, die je nach Massgabe der Karbonathärte des Wassers grösser oder kleiner sein kann und praktisch unkontrollierbar verläuft, wie eigene Versuche bestätigten, wird die elektrochemisch wirksame Oberfläche der Kathode und damit der Stromfluss zwischen den beiden Elektroden verringert.

Anfänglich verwendete TOEDT als Kathodenmaterial Platin, doch zeigte dieses Metall eine derart intensive Kalkfällung, dass brauchbare Messungen auch schon in mittelharten Wässern von 9-12 $2^{\circ}$ frz. H. kaum mehr möglich waren. Mit der Einführung der Goldkathode erzielte er darauf eine wesentliche Verbesserung, indem OHLE innerhalb einer Stunde bei einer Karbonathärte von II, $7^{\circ} \mathrm{frz}$. H. noch keinen merkbaren Stromabfall 
registrierte. Bei Wasser von $2 \mathrm{I}, 2^{\circ}$ frz. $H$. (Plöner Grundwasser) dagegen war eine Messung bereits nicht mehr möglich (OHLE, I953).

Ausser durch den Bikarbonatgehalt scheint die Intensität der Kalkausfällung durch keine anderen Faktoren beeinflusst zu werden. TOEDT (I942) erhielt in Lösungen mit verschiedenem $\mathrm{pH}$ dieselben Resultate, während OHLE (1953) in Wässern mit gleicher Karbonathärte, aber verschiedener Leitfähigkeit, eingestellt mit Kochsalz, denselben zeitlichen Verlauf der Kalkfällung beobachtete.

TOEDT (I954) weist mit Nachdruck darauf hin, dass, neben dem Material selber, seine Oberflächenstruktur von besonderer Bedeutung für die Intensität der Kalkinkrustation ist, und empfiehlt als günstiges Kathodenmaterial ein nach speziellem mechanischem Verfahren geglättetes (mattiertes) Gold.

Noch günstiger ist nach demselben Autor indessen Quecksilber, das infolge seiner amorphen Oberfläche einem Kristallwachstum keine Haftpunkte darbietet. Dafür stösst man bei der Herstellung hydraulisch günstiger Quecksilberkathoden auf Schwierigkeiten, was ihr Verwendungsgebiet auf stationäre Anlagen beschränkt, sei es nun eines der von TOEDT beschriebenen registrierenden Überwachungsgeräte oder eine in irgendwelchen Versuchseinrichtungen eingebaute Messzelle. Dieser Schwierigkeit versuchte man durch Einführung von Amalgamkathoden bestimmter Zusammensetzung aus dem Wege zu gehen, doch haben uns eigene Versuche damit keine wesentlich besseren Resultate ergeben als die reine Goldelektrode. Da zudem die Verformbarkeit von Amalgamen begrenzt und ihre mechanische Stabilität nur gering ist, lassen sich ebenfalls keine hydraulisch günstigen Kathoden herstellen, sondern man hat sich mit der Plattenform zu begnügen.

Wir sind der Ansicht, dass - wenigstens für Messgeräte zum limnochemischen Gebrauch - einer genügenden Strömungsunabbängigkeit mindestens dieselbe Aufmerksamkeit geschenkt werden sollte wie der Kalkindifferenz; es wäre demnach verdienstlich, nach einer Lösung zu suchen, die nicht den einen Faktor auf Kosten des andern eliminiert. Dies scheint uns um so notwendiger, als sich bei kleiner werdender Kathodenfläche der Einfluss des Kalkgehaltes verstärkt, ja so weit gehen kann, dass in hartem Wasser die reproduzierbare Messung mit einer Kleinstkathode nach dem bisher üblichen Messverfahren überhaupt in Frage gestellt wird, wie OHLE an Hand eines bloss II, $7^{\circ}$ harten Wassers zeigte. 


\section{Der zeitliche Stromverlauf bei verschiedenen Elektroden im Langzeitversuch}

In den letzten Kapiteln wurden neben den Grundlagen der elektrochemischen Sauerstoffmessung dicjenigen Fehlerquellen beleuchtet, die grundsätzlich vorhanden sind, also Kalkinkrustation, Strömungs- und Leitfähigkeitsabhängigkeit. Im selben Zusammenhang kamen auch die Massnahmen einer mehr oder weniger vollständigen Elimination dieser Fehler zur Sprache. Man sollte nunmehr annehmen können, dass der elektrochemischen Sauerstoffmessung keine weiteren Hindernisse mehr im Weg stehen - jedenfalls sprechen sich beispielsweise TOEDT und TODT (1953) sehr optimistisch aus: «Es kann abschliessend gesagt werden, dass die elektrochemische Sauerstoffbestimmung von Oberflächengewässern aus dem Versuchsstadium herausgetreten ist.»

Dass indessen unsere nachstehend beschriebenen Versuche einen grösseren Umfang annahmen, als eigentlich beabsichtigt war, gründet auf der Tatsache, dass es schwerhält, eine einfache Einrichtung zu schaffen, die trotzdem alle gestellten Forderungen im praktischen Betrieb wirklich erfüllt.

Wohl besitzen wir in der polarographischen Methode ein nicht zu unterschätzendes Mittel zur·Bestimmung von molekular gelöstem Sauerstoff; die Arbeit mit dem Polarographen indessen ist allgemeinem Gebrauch kaum zugänglich, und zudem sind die notwendigen Einrichtungen recht teuer. Demgegenüber stellt die elektrochemische Sauerstoffmessung eine Methode dar, die infolge ihrer Einfachheit und ihres relativ geringen apparativen Aufwandes unbedingt so weit gebracht werden sollte, dass sie der Gewässerpraxis in die Hand gegeben werden kann. TOEDT hat dieser Entwicklung mit der Konstruktion eines registrierenden Gerätes zur Überwachung von Vorflutern bereits den Weg gewiesen.

So galt es in erster Linie, eine Elektrode zu finden, die in labormässigen Langzeitmessungen über 12 bis 24 Stunden hinweg so konstant arbeitet, dass sie beispielsweise bei Respirationsmessungen an Wassertieren oder in registrierenden Überwachungsanlagen verwendet werden kann. Die zweite, noch in Bearbeitung befindliche Aufgabe besteht in der Konstruktion einer Elektrode, die in Feldmessungen als «Sauerstofflot» eingesetzt werden kann und die neben einer hohen Strömungsunabhängigkeit auch auf Leitfähigkeitsschwankungen möglichst unempfindlich sein muss. Dazu gesellt sich die Forderung nach weitestgehender Linearität der Eichkurven. $\mathrm{Da}$ es sich hier um ausgesprochene Kurzzeitmessungen handelt, fallen da- 
gegen die für die Langzeitmessung charakteristischen Schwierigkeiten teilweise weg.

Bei labormässigen Langzeitmessungen können sämtliche Störfaktoren, wie Temperatur, Leitfähigkeit, Strömungsgeschwindigkeit und eventue'll $\mathrm{pH}$, konstant gehalten werden; unsere Aufmerksamkeit richtet sich demnach neben einer genügenden Sauerstoffempfindlichkeit gänzlich darauf, bei gleichem Sauerstoffgehalt über viele Stunden hinweg gleichstarke, konstante Ströme zu erhalten. Die konstante Strömungsgeschwindigkeit erlaubt zudem die Verwendung hydraulisch ungünstiger Kathoden, beispielsweise Platten oder flüssiges Quecksilber.

In den nachstehenden Versuchen wurden vier Elektrodentypen untersucht:

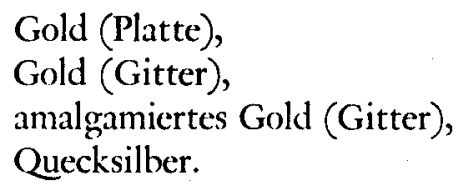

Sämtliche Elektrodengefässe wurden aus Glas hergestellt, da dieses Material ausser seinen guten elektrischen Eigenschaften eine leichte Verformbarkeit besitzt und zudem erlaubt, die auf Lage- und Formänderungen sehr empfindlichen Kathoden stabil einzuschmelzen.

Als Prüfeinrichtung dienten zwei Apparaturen:

a) Umlaufeiurichtung, beschrieben von JAAG (I9SS) (Abb. I). Ein mit konstanter Geschwindigkeit drehender Rührer hält das Wasser in steter, gleichförmiger Bewegung, das infolge seiner periodisch wiederkehrenden Berührung mit der Luft einen sehr konstanten Sauerstoffgehalt besitzt, sofern die Raumtemperatur konstant ist. Ohne zusïtzliche Einrichtungen ist allerdings eine Elektrodenprüfung nur mit sauerstoffgesïttigtem Wasser möglich.

b) Durcblaufapparatur, entwickelt an der Biologischen Abteilung der EAWAG (WUHRMANN und WOKER, I953). Hier wird nicht im Umwälz-, sondern im Durchlaufverfahren gearbeitet, was die Verwendung verschieden sauerstoff haltigen Wassers gestattet, indem ein grösserer, für

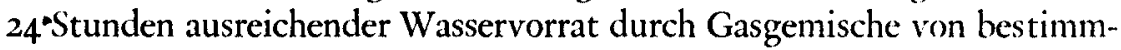
ter Zusammensetzung auf den gewünschten Sauerstoffgehalt gebracht werden kann. Dabei werden die übrigen Komponenten, wie Temperatur und Strömungsgeschwindigkeit, möglichst konstant gehalten und teilweise laufend registriert. Sämtliche Versuche, die wir mit dieser Einrichtung durchführen konnten, wurden mit Hilfe eines Punktschreibers laufend registriert. 
Wir danken an dieser Stelle den Herren Dr. WuHRMANN, Dr. WoKER und W. HeFri für ihre wertvolle Mithilfe.

Während der Messungen gelangten verschiedene Techniken zur Anwendung:

Dauerbelastung. Stromkreis dauernd geschlossen, über Messgerät (Galvanometer) oder über gleichdimensionierten Drahtwiderstand. Ablesung in unregelmässigen Intervallen oder Registrierung.

Intermittierende Belastung. Da in einem Teil der Versuche mit einem Mehrfachschreiber registriert wurde, konnte nur alle 2 Minuten eine Einschaltzeit von 20 Sekunden zur Messung benützt werden. Somit wurde die Elektrode insgesamt während eines Sechstels ihrer gesamten «Laufzeit» belastet.

Sporadiscbe Belastung. Ungefähr alle 60 Minuten eine kurze Belastung von Io Sekunden, dann Ablesung.

Da normalerweise der Sauerstoffdiffusionsstrom im Moment des Einschaltens maximal ist (FrEIER, I952) und in wenigen Sekunden gegen einen bestimmten Grundstrom absinkt, wurde der Strom bei der intermittierenden und der sporadischen Messweise jeweils am Ende der Einschaltzeit (20 bzw. Io Sekunden) abgelesen. Daher dürfen Kurven, die wohl mit derselben Elektrode, jedoch mit verschiedener Messtechnik gewonnen wurden, nicht ohne weiteres miteinander verglichen werden. Dieser Nachteil fällt jedoch ohnehin ausser Betracht, da unsere Problemstellung ja nicht auf Absolutwerte, sondern in erster Linie auf den zeitlichen Verlauf der Kurven ausgerichtet ist.

Um den Einfluss der Kalkinkrustation zum vorneherein auszuschalten, verwendeten wir in einem Teil der Versuche karbonatfreies Wasser, das mit Kochsalz auf eine Leitfähigkeit von $250 \cdot 10^{-6} \Omega^{-1} \cdot \mathrm{cm}^{-1}$ eingestellt worden war. Bei den übrigen Versuchen (Durchlaufapparatur) wurde Leitungswasser mit einer Karbonathärte von is bis $23^{\circ}$ frz. H. verwendet.

\section{Plattenkathode}

Die in Abbildung 2 dargestellte Plattenelektrode wurde bereits früher in Respirationsmessungen an Fliesswassertieren sowie in physikalischen Messungen (JAAG, I955) praktisch erprobt und für solche Zwecke als geeignet befunden, die keine besondere Genauigkeit und keine lange Messdauer erfordern. Ein Versuch, solche Elektrodenpaare bei konstanter Leitfähigkeit und bekannter Geschwindigkeit des Elektrolyten auf Sauerstoff- 
werte zu eichen, scheiterte indessen, da niemals eine wirklich verwendbare Reproduzierbarkeit erreicht wurde. Und dies trotz ausschliesslichem Gebrauch von karbonatfreiem Wasser.

In Abbildung 9 sind die Resultate der Konstanzprüfungen zusammengestellt. Als wesentlichstes Ergebnis geht daraus hervor, dass mit keiner der angemandten Messtecbniken eine genügende Konstanz erreicht werden kann. Während sich intermittierende und sporadische Belastung nur wenig voneinander unterscheiden und fast denselben zeitlichen Verlauf ergeben, bewirkt

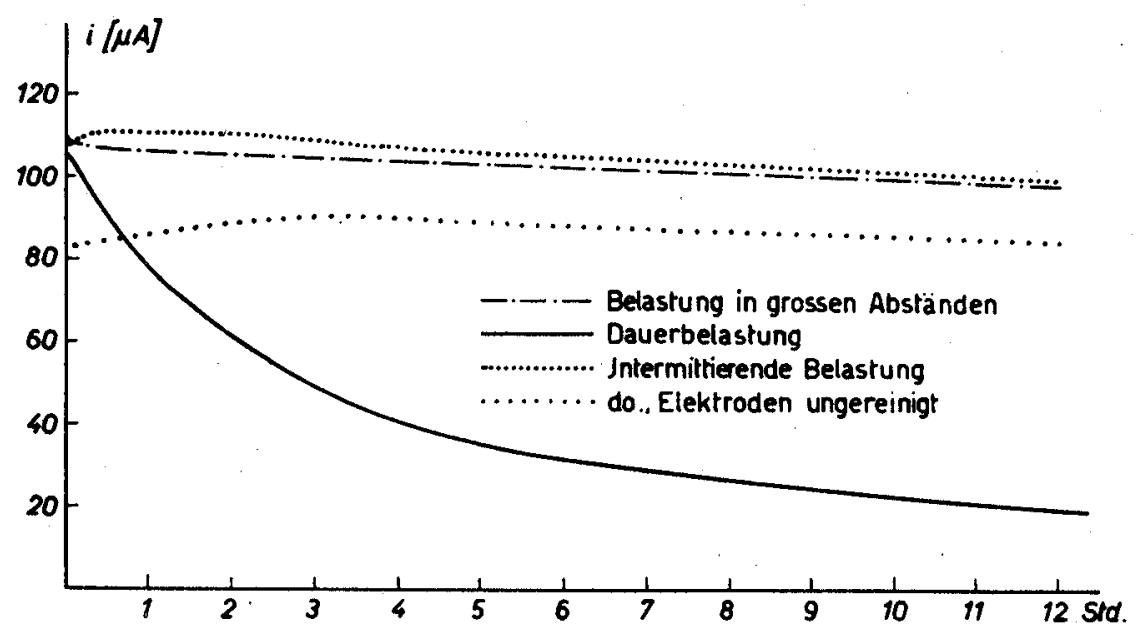

Abb. 9. Verlauf des Diffusionsstroms einer Goldplattenkathode im Langzeitversuch (karbonatfreies, sauerstoffgesättigtes Wasser, $x=250 \cdot 10^{-2} \Omega^{-1} \mathrm{~cm}^{-1}$ ).

Dauerbelastung ein rapides Absinken des Sauerstoffdiffusionsstroms. Nach Absäuern der Elektroden, insbesondere der Kathode, ergibt sich erneut derselbe Verlauf, womit erwiesen ist, dass sich auf der Kathode ein hemmender Belag entwickelt, dessen zeitliche Ausbildung je nach Belastung der Elektroden verschieden ist. Wird der gleiche Versuch mit karbonathaltigem Wasser durchgeführt, so erhält man erwartungsgemäss infolge der zwangsläufigen Kalkabscheidung ein verstärktes Absinken.

Wird das Elektrodenpaar vor Inbetriebnahme nicht abgesäuert bzw. gereinigt, so ergibt sich, wenigstens in den ersten Stunden, ein etwas abweichender Verlauf, indem der Strom vorerst ansteigt, sich mehr oder weniger lang auf einem Maximum hält und dann erst gleichförmig absinkt. Die Höhe dieses Maximalstroms hängt fast ausschliesslich von der seit der letzten Elektrodenreinigung verflossenen Zeit ab. 
Je länger diese "Ruhezeit» ist, um so niedriger sind die Ströme, die nachher gemessen werden. Die Elektroden werden dabei in destilliertem Wasser aufbewahrt; ihr Stromkreis ist nicht geschlossen, so dass sich während dieser Zeit keine elektrochemischen Prozesse zwischen Anode und Kathode abspielen. Beläge von Zinkhydroxyd $\mathrm{Zn}(\mathrm{OH})_{2}$, die sich wolkenartig um die Anode herum ansetzen, sind für den Abfall kaum verantwortlich; jedenfalls ergab ein entsprechender Versuch vor und nach mechanischer Reinigung der Zinkanode dieselben Werte.

Die hemmenden Beläge auf der Kathode wurden nicht weiter auf ihre Beschaffenheit geprüft. Entsprechende Beobachtungen zeigten indessen immer wieder, dass neben elektrochemisch abgeschiedenen Belägen auch Verunreinigungen des Wassers, kleine Fetzchen von Bakterienbelägen, die bei Langzeitversuchen nicht zu verhindern sind, Fasern usw. abgelagert wurden, so dass die zeitliche Inkonstanz des Diffusionsstromes sicherlich nur teilweise auf die vorstehend beschriebenen Erscheinungen zurückzuführen ist, zum andern Teil dagegen durch mechanische Verunreinigungen bewirkt werden. Dazu mag eine Beobachtung von ToeDT und ToDT (1953) interessieren: Die Autoren stellten nämlich bei Langzeitmessungen eine allmähliche Bedeckung der Goldkathode durch eine Schicht von Zinkhydroxyd fest, gefolgt von einem langsamen Absinken des Stromes. Da die Anode die einzig mögliche Quelle einer solchen Verunreinigung sein konnte, wurde sie in der Folge gegen die Kathode durch ein Tondiaphragma abgetrennt, das den Austausch gelöster Stoffe verhindert. Eine ähnliche Anordnung wurde in unseren Versuchen ebenfalls benützt, wobei wir als Diaphragma eine Glassinterplatte verwendeten. Nachdem diese mit einer Pfefferschen Membran imprägniert worden war, zeigte sie hochgradig semipermeable Eigenschaften und trennte während 72 Stunden eine konzentrierte KCl-Lösung, die als Leitelektrolyt für die Anode diente, völlig vom Versuchswasser ab. Als Anode diente dabei ein stabförmiges Stück analysenreines Zink. Konstanzprüfungen derselben Kathode mit offener und abgetrennter Anode zeigten indessen keinen wesentlich verschiedenen Verlauf, so dass wir das Ergebnis ToEDTs, durch die beschriebene Massnahme die allmähliche «Ermüdung» der Goldkathode zu verhindern, nicht bestätigen können.

\section{Gitterkatboden}

Die Gitterelektroden aus reinem oder amalgamiertem Gold wurden mit karbonatfreiem und mit Leitungswasser von etwa $15^{\circ}$ frz. Karbonathärte 
geprüft. Erwartungsgemäss fielen die Leitungswasserversuche insofern negativ aus, als sich hier die Kalkinkrustation entsprechend deutlich auswirkte, und dies nicht nur beim Gold, sondern auch beim Amalgam, wie Abbildung I I zeigt. Doch auch bei Verwendung von karbonatfreiem Wasser konnte keine zeitliche Konstanz festgestellt werden (Abb. 10).

Dass der Messbeginn bei verschiedener Technik verschieden verläuft (steigend bei intermittierender, fallend bei Dauer- und sporadischer Messung), wurde immer wieder beobachtet. Der vorerst stärkere Abfall im

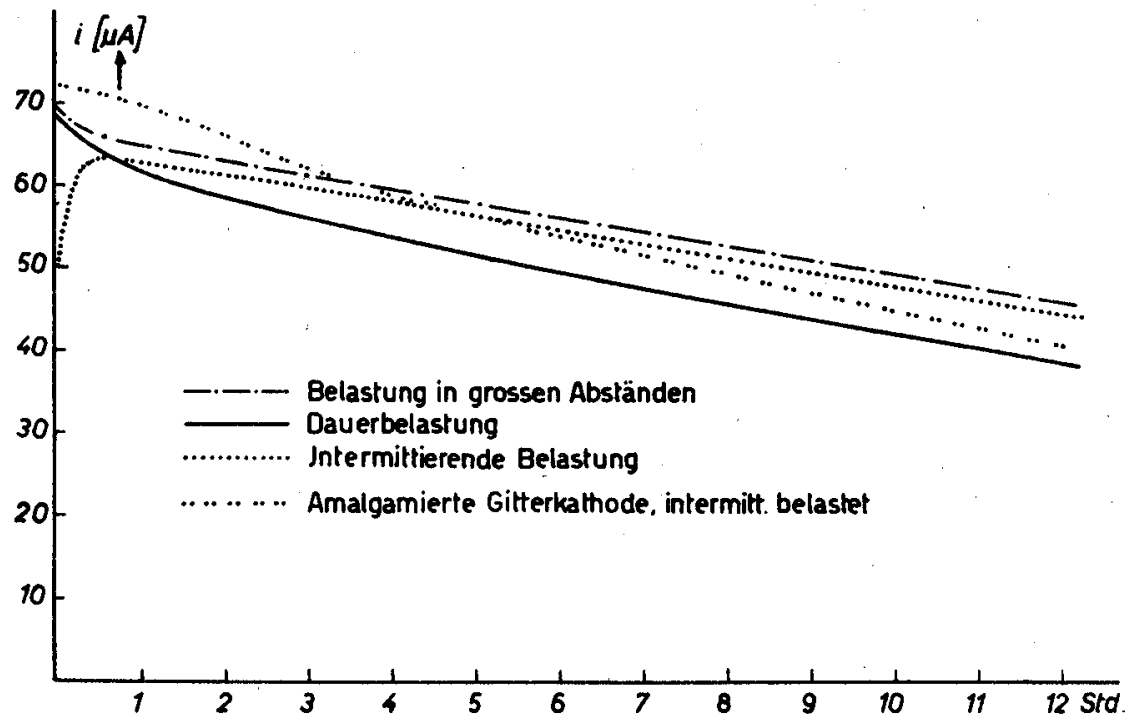

Abb. 10. Verlauf des Diffusionsstroms einer blanken und einer amaigamierten Goldgitterkathode im Langzeitversuch (karbonatfreies Wasser wie Abb. 9).

Daucrbetrieb ist eine bekannte Erscheinung; darum macht sich das gegrenteilige Verhalten bei intermittierender Betriebsweise, wenigstens in karbonatfreiem Wasser, um so augenfälliger bemerkbar. Durch Verwendung verschieden langer Schaltzeiten kann überdies der Verlauf des Anstiẹgs und in gewissem Sinne auch des nachherigen $A b f a l l s$ beeinflusst werden. Diese Erscheinung, deren Ursache uns vorerst unbekannt ist, wird gegenwärtig in unserem Institut weiter verfolgt.

Besonders auffällig ist, dass die mit ein und derselben Elcktrode aufgenommenen Kurven trotz verschiedener Betriebsweise fast völlig parallel laufen. Dass dauernde und intermittierende Belastung ungefähr denselben 
Effekt zeigen, wäre einigermassen verständlich; dass hingegen dic sporadische Betriebsweise, die infolge ihrer geringen relativen Belastungszeit praktisch einer völligen "Ruhe» gleichgesetzt werden kinn, chenfalls den gleichen zeitlichen Verlauf ergibt, beweist, dass der heohachtete Ahfall des Stromes nicht allein auf rein elektrochemische Prozesse zurückgefüht werden kann, sondern dass hier äussere Faktoren mitspiclen, die mit cinfachen Mass-

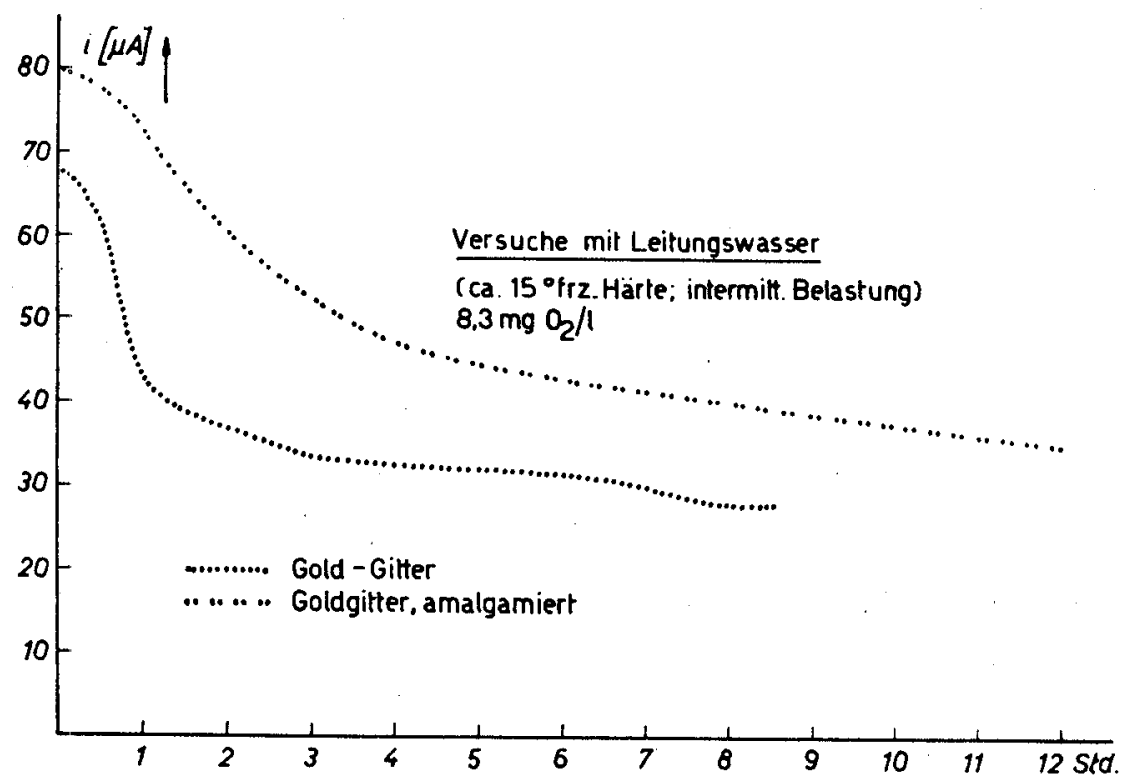

Abb. 11. Diffusionsstrom einer blanken und einer amalganierten (ioldyitterkithode bej Verwendung von Leitungswasser.

nahmen nicht zu eliminieren sind. Namentlich scheint es deshall kaum möglich, mit einer festen Katbode, bestehe sie aus Platin, Gold oder einem .Amalgam, in Langzeitmessungen mirklich konstante und damit reproduzierbare W' erte zu erhalten.

\section{Quecksil berkathoden}

Die einzige Möglichkeit, während langandauernder Messungen zuverlässige Werte zu erhalten, ist die Verwendung von Quecksilber als Kathodenmaterial. Dieses Metall, seit Jahren in der Polarographie als Elektrode verwendet (Tropfelektrode) und von ToEDT erstmals zur elektrochemischen Sawerstoffbestimmung gebraucht, besitzt infolge seines flüssigen 
Zustandes und seiner amorphen Oberfläche denkbar günstige Eigenschaften für seine Verwendung als Kathode. Dafür treten technische Probleme auf. So scheidet die Quecksilberkathode unseres Erachtens infolge ihrer mechanischen Labilität für Feldmessungen ("Sauerstoffsonde») zum vorneherein aus.

Wir verwendeten anfünglich ein näpfchenförmiges Elektrodengefäss aus Glas mit eingeschmolzenem Platindraht. Das Wasser wurde durch einen eingebauten Schnabel direkt auf die Quecksilberoberflüche geleitet; die

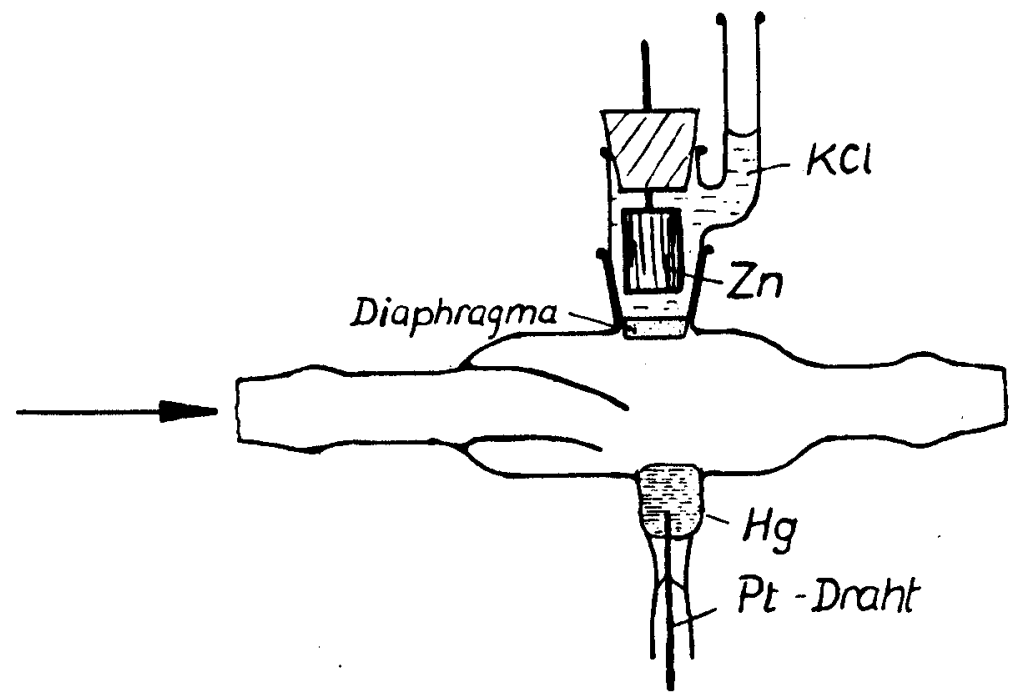

Abb. 12. Elektrodengefäss zur Verwendung von Quecksilber als Kathodenmaterial. Unten: Kathode; oben: Zinkanode mit Diaphragma. Einführung mit Normalschliff.

Anode befand sich dieser gegenüber (Abb. 12). Sämtliche Versuche mit Quecksilber wurden mit Leitungswasser (Karbonathärte $15-23^{\circ}$ frz. H.) durchgeführt, da auf Grund der Toedtschen Ergebnisse anzunehmen war, dass sich der abgeschiedene Kalk nicht störend auf den Diffusionsstrom auswirkte, eine Meinung, die sich allerdings in der Folge als trügerisch erwies.

Abbildung I 3 zeigt den Stromverlauf bei dauernder und intermittierender Belastung. Wie aus diesen Kurven hervorgeht, ist es auch hier unter den gegebenen Umständen nicht möglich, reproduzierbare Werte zu erhalten. Schon bald nach Versuchsbeginn bedeckt sich die anfänglich glänzende Quecksilberoberfläche mit einer blinden, grauen Schicht, die 
sich mehr und mehr verstärkt. Nach einer Versuchsdauer von 24 und mehr Stunden kann diese Schicht als eigentlicher Kalkscherben, durchsetzt mit Quecksilberoxyd und Verunreinigungen aus dem Wasser, entfernt werden. Nach Erneuerung des Quecksilbers wird annähernd der anfänglich erhaltene Wert gemessen, womit auch hier bestätigt ist, dass vornehmlich die $B e-$ deckung bzw. Verunreinigung der Katbode für den Stromabfall verantwortlich ist. Da sich indessen eine Quecksilberoberfläche auf einfache Weise reinhalten lässt, prüften wir die Frage nach einer Elektrodenanordnung, die

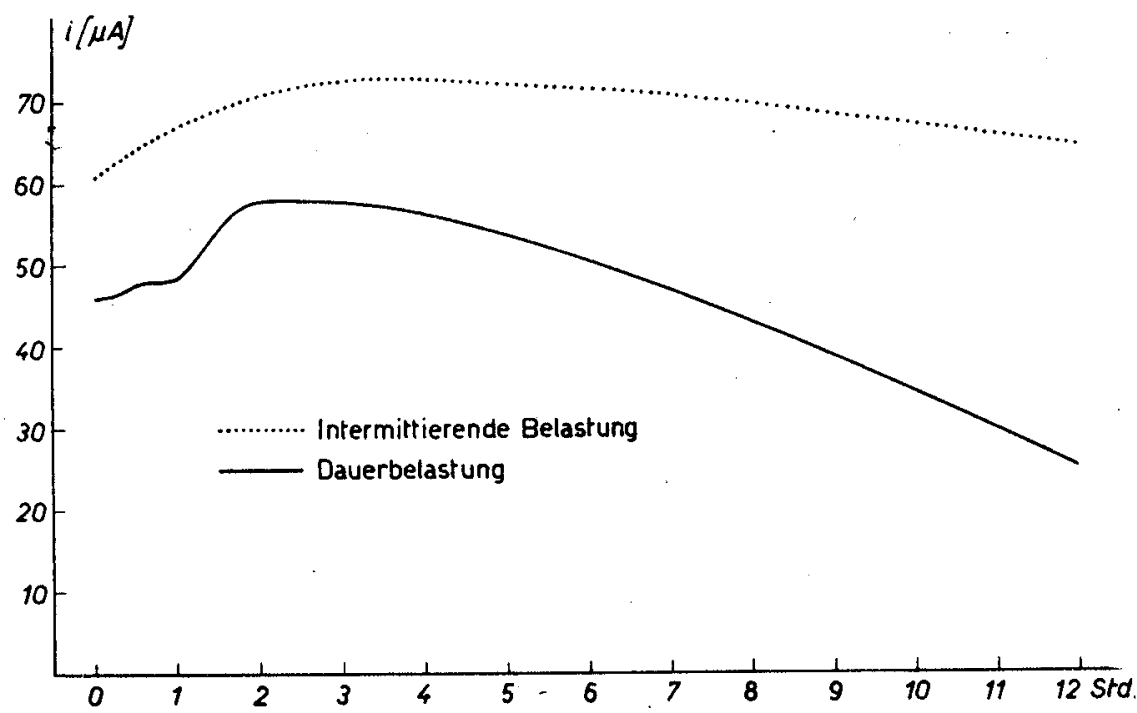

Abb. 13. Diffusionsstrom der Quecksilberkathode bei intermittierender und bei Dauerbelastung.

sich periodisch selbst reinigt, und konstruierten eine Messzelle, wie sie in Abbildung I4 dargestellt ist.

Mit Hilfe eines Synchronmotors wird alle to Minuten ein Schalter betätigt, der einen zweiten, mit der Welle $W$ gekuppelten Motor auslöst. Eine Nockensteuerung, die auf derselben Welle angebracht ist, schaltet ihn nach einer einzigen Umdrehung wieder aus, so dass dic Gummifahne $F$ schliesslich in Abständen von Io zu ro Minuten jeweils innerhalb weniger Sekunden einmal über die Quecksilberoberfläche-hinwegstreicht, dabei teilweise eintaucht und so alle Unreinheiten gewissermassen «abrahmt». Dabei muss sorgfältig darauf geachtet werden, dass die Fahne den Rand des Quecksilbergefässes stets beidseitig berührt und so den Film, der auf 

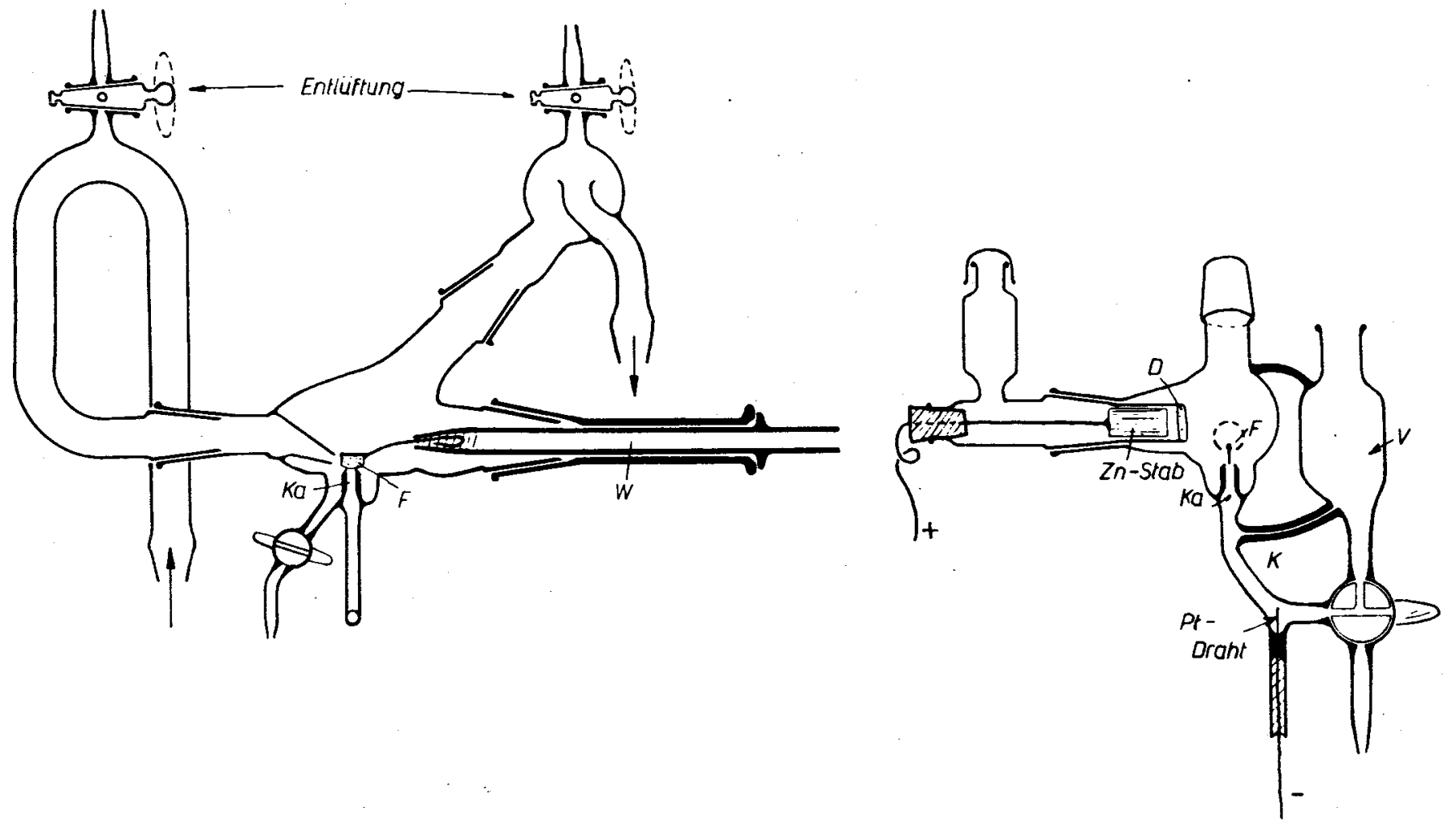
sind auf dem Längsschnitt weggelassen, ebenso die Niveaurohre auf dem Querschnitt. Weitere Erläuterungen im Text. 
der Kathodenoberfläche liegt, vor sich her stösst. Sollte dagegen die Fahne nur einseitig berihbren, so ist der Reinigungseffekt unvollstïndig und unkontrollierbar. In diesem Fall wird der Film nümlich nicht zusammengestossen und entfernt, sondern nur zerrissen, da zwischen Fahne und Gefïss cine Jücke besteht, durch die er ausweichen und sich sofort wieder auf die ganze Oberflïche ausctehnen kann, was infolge der grossen Oberflïchenspannung des Quecksilbers sehr rasch vor sich geht.

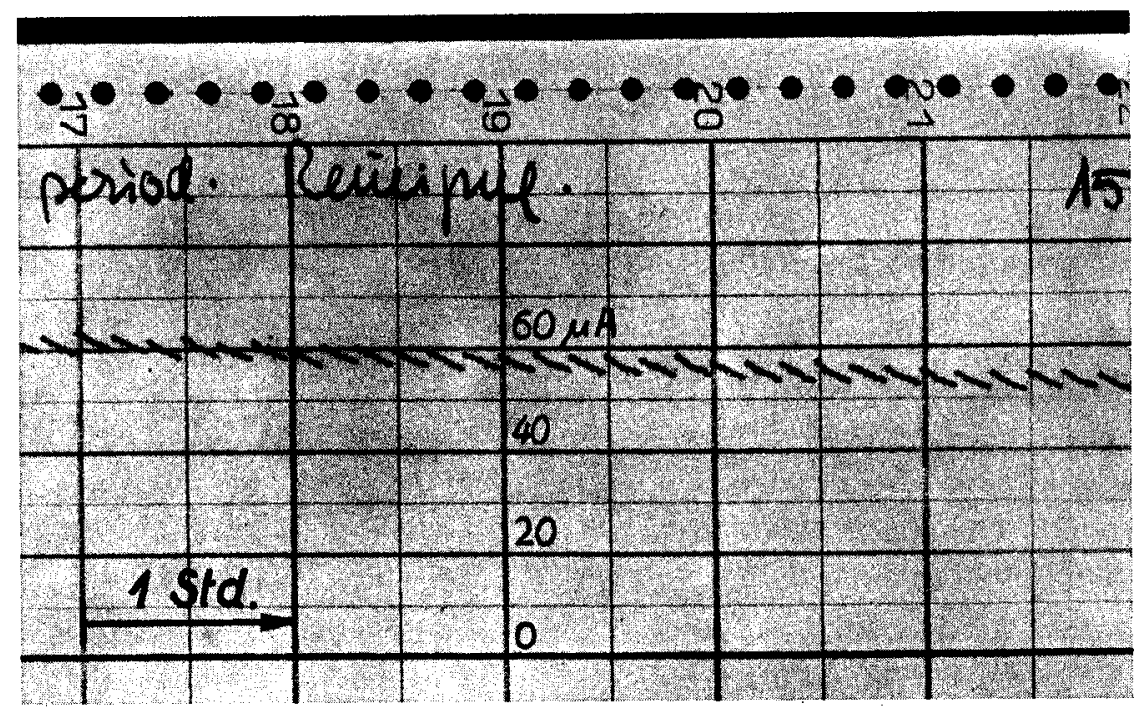

Abb. 15. Versuch Abbildung 16, Kurve a. Ausschnitt aus dem Registrierstreifen. Kathodenreinigung in Abständen von $10^{\circ}$ Minuten mit deutlichem Reinigungseffekt. Der Abfall des Kurvenmittels ist auf die Anode zurückzuführen.

Um einen eventuellen Verlust wieder auszugleichen, enthält ein Vorratsgefäss $I$ eine gewisse Menge Quecksilber, das über eine feine Kapillare $K$ mit dem Kathodengefüss $K_{i l}$ verbunden ist. Diese lediglich kapillare Verbindung wurde deshalb so gewählt, weil bei weitlumigen Röhren oder gar Schliuchen das ganze System in Schwingung geraten kann und dann völlig unbrauchbare Werte liefert. Der Ausgleich über ein sehr enges Rohr dagegen geht gedämpft vor sich und verhindert periodische Schwingungen. Damit aber trotzdem bei Versuchsbeginn dic Niveaux rasch eingestellt werden können, wurde eine zweite, diesmal weitlumige Verbindung mit Hahn vorgesehen, die nach Finspielen des ganzen Systems unterbrochen wird. 
Damit der Wasserdruck auf die Quecksilbersäule konstant bleibt, wurden Ein- und Auslaufteil des Elektrodengefässes mit besonderen Überläufen versehen. Wechselt nämlich die Höhe der Wassersäule, so verändert sich die Höhe des Quecksilbers ebenfalls nach dem Prinzip der kommunizierenden Röhren, und die Folge davon ist in jedem Fall eine Veränderung der Kathodenoberfläche. Durch die beschriebene Anordnung werden indessen solche Schwankungen vermieden.

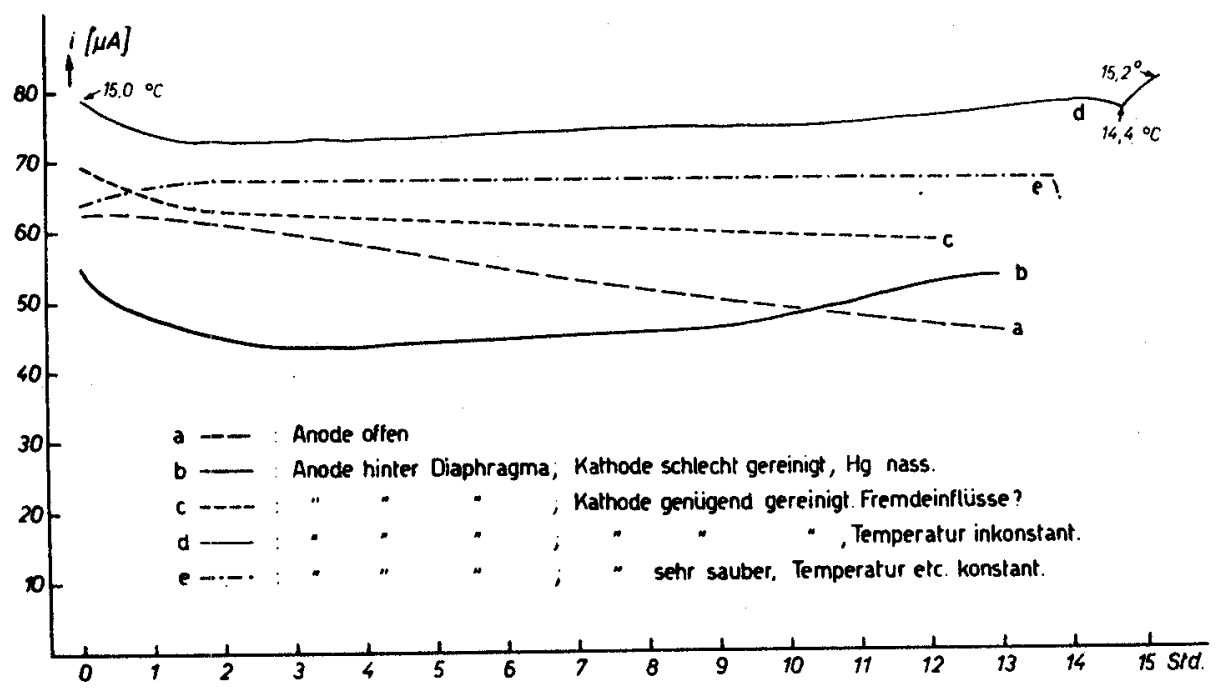

Abb. 16. Zeitlicher Verlauf des Diffusionsstroms einer selbstreinigenden Quecksilberkathode. Einzelheiten siehe Text.

Die Erfordernis eines maximalen Reinigungseffektes macht es weiter notwendig, dass der Durchgestaltung des Quecksilbernapfes grosse Aufmerksamkeit geschenkt wird. So ist es jedenfalls notwendig, den Rand des Gefässes plan zu schleifen und möglichst horizontal zu stellen, sofern man nicht die Möglichkeit hat, den Rand von aussen her anzuschrägen, was das Einhalten einer konstanten Quecksilberkuppe erleichtert. In unseren Anordnungen wurde stets, wie früher schon erwähnt, mit Glas gearbeitet; es wäre indessen denkbar, dass die Verwendung von Plexiglas, das sich leicht maschinell bearbeiten lässt, für die feinere Ausgestaltung des Kathodengefässes gewisse Vorteile bieten würde.

Die Messungen erfolgten normalerweise nicht im intermittierenden, 
sondern im Dauerbetrieb, da wir bei diesen Prüfungen Wert darauf legten, möglichst ungünstige Bedingungen zu schaffen.

Bei den ersten Versuchen diente als Anode ein stabförmiges Stück aus reinstem Zink, das an einem isolierten Draht frei in das Elektrodengefäss hineinragte. Wie nun ein erstes Ergebnis mit einer solchen Anordnung zeigt (Abb. IS), ist ein deutlicher "Reinigungseffekt» vorhanden; nach je Io Minuten wird ein etwas höherer Wert registriert, der ziemlich rasch abfällt und im gesamten Verlauf eine «Sägekurve» ergibt. Trotzdem fällt das Kurvenmittel kontinuierlich ab, obwohl alle Störfaktoren praktisch konstant' geblieben waren (Abb. I6, Kurve $a$ ).

Aus dem Vorhandensein eines deutlichen Reinigungseffektes darf man schliessen, dass die Oberflächenbedeckung der Kathode, bis jetzt die Hauptursache des Stromabfalles, eliminiert ist. Der trotzdem vorbandene Abfall des Kurpenmittels kann somit wur woch mit einer zeitlich fortschreitenden Veränderung der Anode erklärt merden.

Wir versuchten deshalb, auch die Verhältnisse um die Anode möglichst ideal und konstant $z u$ gestalten, indem wir in Anlehnung an TOEDT und TODT (1953) den Zinkteil durch ein Diaphragma $D$ von der Kathode abschlossen. Da sich, wie die Autoren nachwiesen, im Verlaufe einer längeren Messung das Potential des Zinks allmählich veredelt, das heisst, da sich die Anode mit einer Oxydschicht bedeckt, die ein edleres Potential als das Grundmetall aufweist, ist es leicht verständlich, dass eine gleichbleibende Messung ohne besondere Massnahme nicht möglich ist. TOEDT misst allerdings dieser Erscheinung nur eine nebensächliche Bedeutung zu, was wir auf Grund der obigen Resultate jedoch nicht unterstützen können. Bei Kurzzeitmessungen, die sich nur über wenige Minuten erstrecken, mag die Vernachlässigung des Anodenpotentials wohl angehen, da sich hier Verschiebungen kaum spürbar manifestieren. Geht es indessen um Langzeitmessungen, und dazu zählen wir auch ganz besonders registrierende Überwachungsanlagen, so muss neben der Kathode auch der Anode genügende. Aufmerksamkeit geschenkt werden.

Die Bauweise des Anodenteils wurde aus den früheren Versuchen (siehe Abb. I2) übernommen. Eine Glasfritte feinster Körnung, mit einer Pfefferschen Membran imprägniert, wurde in ein Glasrohr mit Normalschliff eingeschmolzen; ein angesetztes Vorratsgefäss diente zur Aufnahme eines Überschusses an konzentrierter Kaliumchloridlösung (Abb. 14). Die eigentliche Anode, ein stabförmiges Zinkstück von Analysenqualität, wurde vor Inbetriebnahme kräftig mit Salzsäure gebeizt, währenddem der Draht, an dem das Zink befestigt ist; gegen die Flüssigkeit isoliert ist. 
Dass bei ähnlichen Versuchen mit Goldkathoden (S. 140) mit und ohne Diaphragmaanode kein wesentlicher Unterschied in den zeitlichen Stromverlaufskurven festgestellt wurde, hat verschiedene Gründe. Einerseits wurde beobachtet, dass der zeitliche Abfall des Sauerstoff-Diffusionsstroms ziemlich unkontrollierbar verläuft, und anderseits scheint die allmähliche Bedeckung der Anode gegenüber derjenigen der Kathode so gering zu sein, dass sie im gesamten nicht mehr deutlich zur Geltung kommen konnte. Da hingegen bei der selbstreinigenden Quecksilberkathode die Kathodenbedeckung ausfällt, kommt hier der eigentliche Anodeneffekt allein zum Ausdruck.

Der erste Versuch, der mit dieser Einrichtung durchgeführt wurde, ergab noch kein befriedigendes Resultat, da infolge eines Betriebsunfalles die Quecksilberoberfläche nur unvollständig gereinigt wurde und zudem die Verbindungskapillare zwischen Kathodennapf und Vorratsgefäss durch einen Wassereinbruch verunreinigt worden war, so dass kein Ausgleich mehr stattfinden konnte. Trotzdem sinkt der Strom nur in den ersten 3 Stunden, bleibt dann während etwa 4 Stunden einigermassen konstant und beginnt zuletzt anzusteigen (Abb. 16, Kurve $b$ ). Der Reinigungseffekt (Sägekurve) zeigt sich deutlich über den ganzen Versuch hinweg.

Im Hinblick auf eine optimale Reinigung wurde vor dem zweiten Versuch der Reinigungsmechanismus neu justiert sowie die Gummifahne leicht mit Vaseline eingefettet, da sich dadurch das Kalkhäutchen leichter entfernen lässt. Der Versuch selber, wiederum unter Dauerbelastung der Elektroden durchgeführt, ergab den aus Kurve $c$ in Abbildung I6 ersichtlichen Stromverlauf. Dabei verläuft das Kurvenmittel noch nicht völlig horizontal, doch ist der Abfall sehr gering und möglicherweise auf Fremdeinflüsse, wie Schwankungen in der Temperatur oder der Leitfähigkeit, zurïckzuführen.

Ein dritter Versuch, wiederum unter Berücksichtigung aller Vorsichtsmassnahmen angesetzt, ergab im Gegensatz dazu eine erst fallende, dann aber sehr langsam ansteigende Kurve mit kleineren Schwankungen (Abb. 16, Kurve $d$ ). Es stellte sich indessen heraus, dass infolge einer Störung an der Klimaanlage die Temperatur des Wasserbades nicht konstant geblieben war. So betrug sie am Anfang $15,0^{\circ} \mathrm{C}$ bei einem Strom von $79 \mu \mathrm{A}$, während wir am Ende der Messung, also 14 Stunden später, eine Temperatur von $14,4^{\circ} \mathrm{C}$ bei einem Strom von $76 \mu \mathrm{A}$ feststellten. Eine rasche Erwärmung auf $15,2^{\circ} \mathrm{C}$ wurde gefolgt von einem Stromanstieg um $5 \mu \mathrm{A}$ auf $8 \mathrm{I} \mu \mathrm{A}$. Somit dürften die festgestellten Schwankungen innerhalb des Versuches auf Temperaturänderungen zurückzuführen sein. 
Diese Resultate liessen es als wünschbar erscheinen, eine weitere Konstanzprüfung unter solchen Bedingungen durchzuführen, wie sie in der Versuchspraxis angewandt werden, also im intermittierenden Betrieb unter genauester Konstanthaltung von Leitfähigkeit, Durchfluss, Temperatur usw., wozu der an der Biologischen Abteilung der EAWAG installierte Brown-Honeywell-Schreiber benützt werden konnte. Dieses Gerät gestattete, neben dem Sauerstoff-Diffusionsstrom gleichzeitig den $\mathrm{pH}$ Wert und die Temperatur des Versuchswassers zu registrieren.

Gearbeitet wurde mit einem Messrhythmus von I44 Sekunden; nach Ablauf ron 126 Sekunden schaltete sich jeweils die mit der Hg-Zn-Elek-

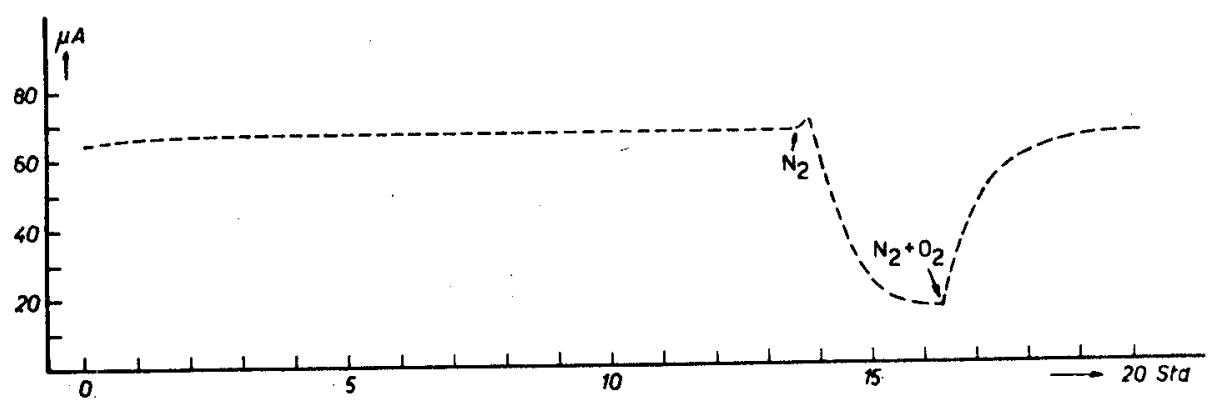

Abb. 17. Stromverlauf einer selbstreinigenden Quecksilberkathode bei optimaler Reinigung und völlig konstanten sonstigen Bedingungen. Zudosierung von Stickstoff bewirkt entsprechende Verminderung, Zugabe von Sauerstoff Erhöhung des Diffusionsstroms (Kurve $e$ in Abb. 16: stammt aus dem gleichen Versuch).

trode belegte Meßstelle für 18 Sekunden ein und stempelte am Ende dieser Zeit den erreichten Messwert auf den Registrierstreifen.

In Abbildung 17 ist der zeitliche Verlauf des Sauerstoff-Diffusionsstromes während des 20 Stunden dauernden Versuches dargestellt (Kurve $e$ in $\mathrm{Abb}$. I 6 bezieht sich auf den gleichen Versuch; sie wurde dort lediglich aus Vergleichsgründen wiedergegeben). Nachdem sich nach ungefähr 2 Stunden der Sauerstoffgehalt des Wassers auf rund $10 \mathrm{mg} \mathrm{O}_{2} / 1$ eingespielt hatte, blieb das Kurvenmittel wäbrend 12 Stunden pöllig konstant. Der Reinigungseffekt kommt auch hier, beim intermittierenden Betrieb, über den gesamten Verlauf hinweg deutlich zum Ausdruck. Auf Veränderungen des Sauerstoffgehaltes durch Einleitung von Gasgemischen verschiedener $\mathrm{Zu}$ sammensetzung reagierte die Elektrode unverzüglich (Abb. 17). Der unmittelbar àn den fast vierzehnstündigen Konstanzversuch anschliessende Abfall ist das Resultat einer Zudosierung von reinstem Stickstoff, während 
der darauffolgende Anstieg durch Einleiten eines Gemisches von Stickstoff und Sauerstoff bewirkt wurde. Der $A$ bfall von rund io auf $1,5 \mathrm{mg} \mathrm{O} \mathrm{O}_{2} / 1$ entspricht einer Stromverminderung von 67 auf $16,5 \mu \mathrm{A}$.

Da sich bei Messungen mit hohen Anforderungen an die Genauigkeit die sogenannte Sägekurve störend bemerkbar machen könnte, wurde das Vorgelege der Schaltuhr, die den Reinigungsmechanismus auslöst, so umgebaut, dass nach jeder Messung, somit nach je I44 Sekunden, eine Reini-

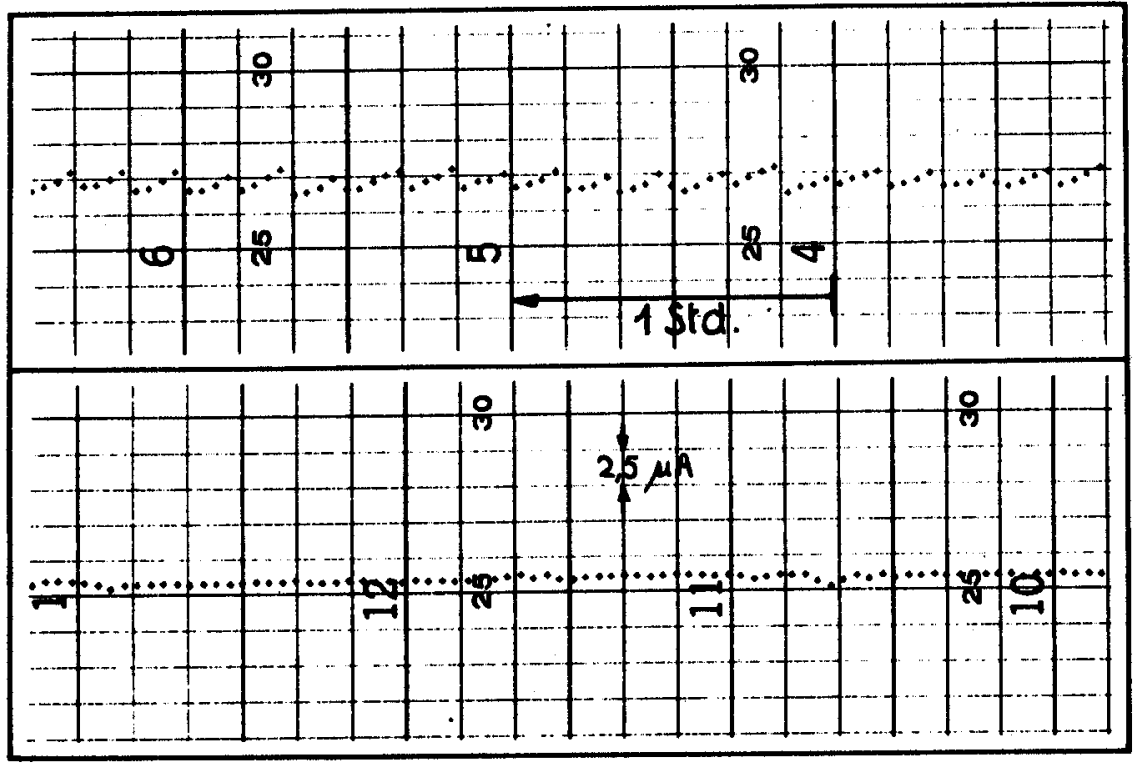

Abb. 18. Oben : Versuch Abbildung 17, Ausschnitt aus dem Registrierstreifen. Kathodenreinigung in Abständen von 10 Minuten; der Reinigungseffekt ist deutlich erkennbar. - Unten:

Reinigung der Kathode nach jedem Messpunkt, das heisst nach je 144 Sekunden.

gung erfolgt. Während vorher nur alle Io Minuten gereinigt wurde, wird nun jeder Registrierpunkt unter genau denselben Bedingungen erzeugt, wodurch eine zusammenhängende gerade Linie entsteht, deren Verlauf auch feinere Schwankungen erkennen lässt (Abb. I8).

Auf Grund dieser Ergebnisse sind wir somit beute in der Lage, den Sauerstoffgehalt eines Wassers im Durcblaufversuch mit der elektrochemischen Methode zuperlässig registrierend zu verfolgen, sofern die übrigen Faktoren, mie Durchfluss, Temperatur und Salzgebalt, konstant bleiben.

Uber die erreichbare Genauigkeit soll in einer späteren Mitteilung ausführlich berichtet werden. 
Die vorliegende Studie bezweckte vor allen Dingen, die praktische Anwendbarkeit der elektrochemischen Sauerstoffbestimmung zu prüfen und kritisch zu beleuchten. Die anfänglich sehr wenig ermutigenden Resultate, die mit den Angaben in der Literatur in keinen richtigen Einklang zu bringen waren, konnten aber im Laufe einer längeren Entwicklung durch den Einsatz verschiedener Elektrodentypen soweit verbessert werden, dass wir heute in der Lage sind, vorerst wenigstens den Sauterstoffgehalt eines sonst konstanten Wassers, wie es innerhalb eines Versuches zur Anwendung gelangen mag, dauernd genau $\mathrm{zu}$ messen und $\mathrm{zu}$ registrieren. Die Methode soll nun in nächster Zeit weiter ausgebaut werden. So sind beispielsweise Verfeinerungen in der Gestaltung des Elektrodengefässes sowie einfache Zusatzeinrichtungen vorgesehen, die es ermöglichen sollen, auch in Wässern von wechselnder Qualität, namentlich in Abwasser und Flusswasser, den Sauerstoffgehalt zu registrieren.

Wir sehen der künftigen Entwicklung der elektrochemischen Sauerstoffmessung zuversichtlich entgegen. Wir möchten aber vorderhand vor einem zu grossen Optimismus warnen, da noch mannigfaltige Schwierigkeiten methodischer Art zu überwinden sind, sofern man an die Reproduzierbarkeit der Messwerte einige Anforderungen stellt. Wohl zweifeln wir nicht daran, dass über kurz oder lang ein Gerät vorliegen wird, das eine zuverlässige und genaue Vorflut- oder Abwasserüberwachung auf elektrochemischer Grundlage erlaubt und das ausserdem zu einem erschwinglichen Preis erhältlich sein wird, und wir hoffen auch, in absehbarer Zeit eine Sauerstoffsonde für limnologischen Gebrauch zu besitzen, die nach den hier zusammengestellten Erkenntnissen gebaut wurde; entsprechende Voruntersuchungen liegen bereits vor. Aber wir möchten damit der Meinung entgegentreten, die elektrochemische Sauerstoffmessung liege bereits als ausgereifte Methode zum Gebrauch bereit. Bevor die praktische Anwendung, beispielsweise im Sinne einer Weiterentwicklung der von OHLE (1953) und von TOEDT und Mitarbeitern (1953; 1955) beschriebenen Sauerstoffsonden, endgültig an die Hand genommen werden kann, ist es unumgänglich notwendig, die methodischen Grundlagen so weitgehend wie möglich abzuklären.

\section{ZUSAMMENFASSUNG}

I. Die vorliegende Arbeit behandelt neben den theoretischen Grundlagen vor allem die praktischen Fragen, die sich aus der Anwendung der elektrochemischen Sauerstoff bestimmung ergeben. 
2. Versuche, die durch die Prandtlsche Grenzschicht hervorgerufene Strömungsabhängigkeit des Sauerstoff-Diffusionsstroms zu verringern, führten zur Konstruktion gitterförmiger Kathoden aus Gold. Diese sind in hydraulischer Hinsicht optimal und können auch als Kleinstkathoden gebaut werden.

3. Der Einfluss von Leitfähigkeitsschwankungen des Elektrolyten auf den Diffusionsstrom kann nicht völlig ausgeschaltet werden, indem die entsprechenden Leitfähigkeits-Strom-Kurven erst bei hohen Salzgehalten achsenparallel laufen. Indessen kann er durch Verkleinerung der Kathode immerhin bedeutend abgeschwächt werden.

4. Die Kalkinkrustation auf der Kathode, die in bikarbonathaltigem Wasser eine Messung erschwert oder gar verunmöglicht, konnte auch durch die Verwendung von Gold-Amalgam-Kathoden nach TOEDT (1954) nicht eliminiert werden.

5. Die elektrochemische Sauerstoffmessung über längere Zeit hinweg bietet besondere Schwierigkeiten, da auch unter konstanten Bedingungen normalerweise keine gleichbleibønden Ströme zu beobachten sind.

6. Langzeitversuche mit karbonatfreiem und karbonathaltigem Wasser ergaben, dass Goldplatten-, Goldgitter-, Goldamalgamgitter- und Quecksilberkathoden inkonstante Werte lieferten. Sämtliche Elektroden, die zudem mit verschiedenen zeitlichen Schaltintervallen belastet wurden, lieferten stets einen Strom, der sich mit zunehmender Zeit verringerte. Die Verwendung einer Zinkanode hinter einem Diaphragma ergab keine deutliche Verbesserung.

7. Eine selbstreinigende Quecksilberkathode, die in Abständen von wenigen Minuten durch eine motorgetriebene Gummifahne dauernd sauber gehalten wird, ergab einen beinahe konstanten Strom. Der noch vorhandene zeitliche Stromabfall konnte durch Verwendung einer Zinkanode hinter einem Diaphragma vollständig eliminiert werden. Durch kürzere Zeitintervalle zwischen den' einzelnen Reinigungen wurde auf einem Punktschreiber eine praktisch gerade Linie erhalten.

8. Es ist somit möglich, den Sauerstoffgehalt eines in bezug auf Leitfähigkeit, Temperatur und Durchflussgeschwindigkeit konstanten Wassers während beliebig langer Zeit zu registrieren.

9. Die selbstreinigende Hg-Elektrode wird zur Zeit weiter ausgebaut im Hinblick auf eine allgemeine Anwendbarkeit in Laboratorium- und Gewässerpraxis. Eine verbesserte Sauerstoffsonde für feldmässige Kurzzeitmessungen befindet sich ebenfalls in Vorbereitung. 


\section{SUMMARY}

I. The foregoing paper deals with the theoretical aspects and especially with the practical questions which arise in the application of the electrochemical oxygen determination.

2. In order to decrease the influence of the water flow upon the oxygen diffusion current gold screen cathodes were found to be most suitable. Such screen eathodes response to their hydraulic behaviour very favourably. They also can be built conveniently as micro cathods.

3. It has been hardly possible to eliminate the influence of irregular fluctuations of the clectrolytic conductivity upon the diffusion current. The current-conductivity curves present constant current values at relatively high salt concentrations. The corresponding conductivities at a constant current show the following values: $800 \mathrm{ppm} \mathrm{NaClf} x=1500 \cdot 10^{-6} \Omega^{-1} \cdot \mathrm{cm}^{-1} ; 1200 \mathrm{ppm} \mathrm{NaCl} / x=2000 \cdot 10^{-6} \Omega^{-1} \mathrm{~cm}^{-1}$.

4. The precipitation of calcium carbonate at the cathode, which occurs in relatively hard water rendering an exact measurement difficult or impossible, could not be avoided even by using gold amalgame cathodes introduced by TOEDT (1954).

5. The electrochemical determination of oxygen offers especial difficulties if the measurement has to be made over a long period (for several hours). Even under constant conditions it is not possible to record constant currents.

6. Investigations over long test periods with soft and hard water show that cathodes made from gold plates, gold screens, gold-amalgame screens and mercury do not yield constant values. All these electrodes produce current densities which decrease with increasing time intervals; no matter whether the circuit is kept open at frequent intervals or kept closed for the entire period ( 12 hours). The use of a diaphragm between the Zinc anode and the cathode did not improve the measurments remarkably.

7. A self-cleaning mercury cathode is constructed. The surface of the mercury is purified by a motor driven rubber spatula which removes periodically all impurities. By using this accessory the influence of time on the current as shown in Figure 16, curve a, becomes smaller and this difference could finally be eliminated by the use of a diaphragm as already mentioned. Use of the cleaning mechanism within shorter time intervals (synchronous with the frequency of the recording instrument) enabled us to record practically constant currents.

8. It is, therefore, possible to determine the oxygen content electrochemically over a very long period only when the conductivity (salinity), the temperature and the circulating velocity of the water remain constant.

9. It is intended to improve further the self-cleaning mercury electrode in order to introduce it as a commonly applicable instrument in laboratory and water works practice. A special apparatus (oxygen sounder) for improved electrochemical oxygen determination in field-work is also in preparation. 


\section{IITERATURVERTEIC:HNIS}

FREIER, R., Neue .Metboden zur Bestimmung des im It asser geliosten Sauerstoffi in Gegenmart reduzierender Stoffe, Jb. "Vom Wasser" 19 (1952).

JAAG, O., Die rërunreinigung pon Fliessgenä̈ssern, dargestellt am Reispiel des Rlcinstromes (München 1954, im Druck).

OHLE, W., Prïfung und Anwendung der elektrocbemischen Sauerstoff bestimmung fïr Gemässeruntersuchungen, Jb. "Vom Wasser" 19 (1952).

()HLE, W., Die chemische und die elektrocbemiscbe Bestimmung des molekular gelisten Sauerstoffs der Binnengemässer, Internat. V'r. Limn. 1953, 3.

PR.ANDTL, L., Strömungslebre (K. Vicweg, Braunschwcig 1942).

TöDT, F., Grundlagen und Anwendung der elektrochemisclen Bestimmung des im I/ asser gi'lïsten Sauerstoffs, Gesundheitsing. 65 (1942).

TÖDT, F, und TODT, H.-G., Die elektrochemische Bestimmung des Sauerstoffgehaltes von Oherflächengewässern, Jb. «Vom Wasser» 20 (1953).

TÖDT, F., SCHWARZ, W., und TODT, H.-G., Llektrochemische Bestimmung des Sauerstoffgebaltes von Oberfläcbengewässern, Gesundheitsing. 75, 13/14 (1954).

TÖDT, F., und PЕтSCH, G., Üher die "Ieiterentmicklung des "Sauerstofflotes» zur elektrochemischen Sofortablesung des in offenen Gemässern gelösten Sauerstoffs, Giesundhcitsing. 76, $7 / 8$ (1955).

TRÜMPLLR, G., und ZELLER, H., Zw' Strömungsabhängigkeit won (Grenzstrïmen, Helv. chim. Acta 34, 952 (1951).

WUHRMANN, K., und WOKER, H., Uber die Giftmirkungen von .tmmoniak- und Zyanidlissungen mit verschiedener Sauerstoffspanmung und Temperatur auf Fische, Schweiz. Z. Hydrol. 15, 2 (1953). 